\title{
Some Propositions on Generalized Nevanlinna Functions of the Class $\mathcal{N}_{k}$
}

\author{
Yan-Ping Song, ${ }^{1,2}$ Hui-Feng Hao, ${ }^{1}$ Yong-Jian Hu, ${ }^{1}$ and Gong-Ning Chen ${ }^{1}$ \\ ${ }^{1}$ School of Mathematical Sciences, Beijing Normal University, Laboratory of Mathematics and Complex Systems, \\ Ministry of Education, Beijing 100875, China \\ ${ }^{2}$ The College of Mathematics and Physics, Xinjiang Agricultural University, Urumqi 830052, China
}

Correspondence should be addressed to Yong-Jian Hu; yongjian@bnu.edu.cn

Received 1 August 2014; Accepted 3 October 2014; Published 23 October 2014

Academic Editor: Mahouton N. Hounkonnou

Copyright (C) 2014 Yan-Ping Song et al. This is an open access article distributed under the Creative Commons Attribution License, which permits unrestricted use, distribution, and reproduction in any medium, provided the original work is properly cited.

\begin{abstract}
Some propositions on the generalized Nevanlinna functions are derived. We indicate mainly that (1) the negative inertia index of a Hermitian generalized Loewner matrix generated by a generalized Nevanlinna function in the class $\mathcal{N}_{\kappa}$ does not exceed $\kappa$. This leads to an equivalent definition of a generalized Nevanlinna function; (2) if a generalized Nevanlinna function in the class $\mathcal{N}_{\kappa}$ has a uniform asymptotic expansion at a real point $\alpha$ or at infinity, then the negative inertia index of the Hankel matrix constructed with the partial coefficients of that asymptotic expansion does not exceed $\kappa$. Also, an explicit formula for the negative index of a real rational function is given by using relations among Loewner, Bézout, and Hankel matrices. These results will provide first tools for the solution of the indefinite truncated moment problems together with the multiple Nevanlinna-Pick interpolation problems in the class $\mathcal{N}_{\kappa}$ based on the so-called Hankel vector approach.
\end{abstract}

\section{Introduction}

Let $\mathbb{C}, \mathbb{R}$, and $\mathbb{N}$ be the complex plane, the real axis in the complex plane, and the set of nonnegative integers, respectively. Denote by $\mathbb{C}^{+}$and $\mathbb{N}^{+}$the open upper half complex plane and the set of positive integers, respectively. For a meromorphic function $f(z)$ of one complex variable, its domain of holomorphy of $f(z)$ is written as $D(f)$. Denote by $v(A)$ the number of negative eigenvalues of a Hermitian matrix $A$.

In 1977, Krein and Langer [1, Section 1] introduced the function class $\mathcal{N}_{\kappa}$ of all generalized Nevanlinna functions with negative index $\kappa \in \mathbb{N}$.

Recall that a meromorphic function $f(z)$ in $\mathbb{C} \backslash \mathbb{R}$ is called a generalized Nevanlinna function with negative index $\kappa \in$ $\mathbb{N}$, if it satisfies the symmetric condition: $f(\bar{z})=\overline{f(z)}$ for all $z \in D(f)$, and, for each choice of $m \in \mathbb{N}^{+}$, distinct points $z_{1}, \ldots, z_{m} \in \mathbb{C}^{+} \cap D(f)$, the Hermitian matrix

$$
P_{f}\left(z_{1}, \ldots, z_{m}\right):=\left(\frac{f\left(z_{i}\right)-\overline{f\left(z_{j}\right)}}{z_{i}-\bar{z}_{j}}\right)_{i, j=1}^{m}
$$

has at most $\kappa$ negative eigenvalues (counted with multiplicities) and for at least one such choice exactly $\kappa$ negative eigenvalues; that is,

$$
\begin{aligned}
\max & \left\{v\left(P_{f}\left(z_{1}, \ldots, z_{m}\right)\right)\right. \\
& \left.\mid z_{1}, \ldots, z_{m} \in \mathbb{C}^{+} \cap D(f), m \in \mathbb{N}^{+}\right\}=\kappa .
\end{aligned}
$$

In particular, for $\kappa=0$, the class $\mathcal{N}_{0}$ is actually the set of all Nevanlinna functions which are holomorphic in $\mathbb{C} \backslash \mathbb{R}$ such that $f(\bar{z})=\overline{f(z)}$ and $\operatorname{Im} f(z) / \operatorname{Im} z \geq 0$ for all $z \in \mathbb{C} \backslash \mathbb{R}$. It is well known that (see, e.g., [2,3]) each $f(z) \in \mathcal{N}_{0}$ admits an integral representation of the form:

$$
\begin{array}{r}
f(z)=\alpha z+\beta+\int_{\mathbb{R}}\left(\frac{1}{u-z}-\frac{u}{1+u^{2}}\right) \mathrm{d} \sigma(u), \\
\forall z \in \mathbb{C} \backslash \mathbb{R},
\end{array}
$$

in which $\alpha \geq 0, \beta \in \mathbb{R}$, and $\sigma(u)$ is a positive measure on $\mathbb{R}$ satisfying

$$
\int_{\mathbb{R}}\left(1+u^{2}\right)^{-1} \mathrm{~d} \sigma(u)<+\infty .
$$


By definition, we check easily that (see, e.g., $[1,4]) f(z) \in$ $\mathcal{N}_{\kappa}$ and $f(z) \not \equiv 0$ if and only if $f\left(-z^{-1}\right) \in \mathcal{N}_{\kappa}$, and also, if and only if $-f(z)^{-1} \in \mathcal{N}_{\kappa}$. Moreover, if $f(z) \in \mathcal{N}_{\kappa_{1}}$ and $g(z) \in \mathcal{N}_{\kappa_{2}}$, then $f(z)+g(z) \in \mathcal{N}_{\kappa}$, where $\kappa \leq \kappa_{1}+\kappa_{2}$.

For $\alpha \in \mathbb{R}$, the symbol $\pi_{\varepsilon}(\alpha)$ stands for the sector in $\mathbb{C}^{+}$ with the vertex $\alpha$ :

$$
\pi_{\varepsilon}(\alpha)=\{z \mid \varepsilon \leq \arg (z-\alpha) \leq \pi-\varepsilon\} \quad\left(0<\varepsilon<\frac{\pi}{2}\right) .
$$

The notation $\angle \lim _{z \rightarrow \alpha} f(z)=A$ means that the function $f$ has a nontangential limit $A$ at $t \in \mathbb{R}$; that is, $f(z)$ tends to $A$ as $z$ tends to $\alpha$ in each sector $\pi_{\varepsilon}(\alpha)$. Also, the notation $\angle \lim _{z \rightarrow \infty} f(z)=A$ stands for the nontangential limit of $f$ as $z \rightarrow \infty$ in each sector $\pi_{\varepsilon}(0)$.

Now, we introduce the notions of generalized zeros and generalized poles of nonpositive type of a generalized Nevanlinna function $f(z) \in \mathscr{N}_{\kappa}$ (see [5-8] for details).

A point $\alpha \in \mathbb{R}$ is called a generalized zero of nonpositive type of multiplicity $\pi$ of $f(z) \in \mathcal{N}_{\kappa}$ if

$$
\begin{aligned}
& -\infty<\angle \lim _{z \rightarrow \alpha}(z-\alpha)^{-2 \pi+1} f(z) \leq 0, \\
& 0<\angle \lim _{z \rightarrow \alpha}(z-\alpha)^{-2 \pi-1} f(z) \leq+\infty .
\end{aligned}
$$

Similarly, the point $\infty$ is called a generalized zero of nonpositive type of multiplicity $\pi$ of $f(z) \in \mathcal{N}_{\kappa}$ if

$$
\begin{aligned}
& 0 \leq \angle \lim _{z \rightarrow \infty} z^{2 \pi-1} f(z)<+\infty \\
& -\infty \leq \angle \lim _{z \rightarrow \infty} z^{2 \pi+1} f(z)<0 .
\end{aligned}
$$

A point $\beta \in \mathbb{R}$ is called a generalized pole of nonpositive type of multiplicity $\pi$ of $f(z) \in \mathcal{N}_{\kappa}$ if

$$
\begin{gathered}
0<\angle \lim _{z \rightarrow \beta}(z-\beta)^{2 \pi-1} f(z) \leq+\infty, \\
-\infty<\angle \lim _{z \rightarrow \beta}(z-\beta)^{2 \pi+1} f(z) \leq 0 .
\end{gathered}
$$

Similarly, the point $\infty$ is called a generalized pole of nonpositive type of multiplicity $\pi$ of $f(z) \in \mathcal{N}_{\kappa}$ if

$$
\begin{aligned}
& -\infty \leq \angle \lim _{z \rightarrow \infty} z^{-2 \pi+1} f(z)<0, \\
& 0 \leq \angle \lim _{z \rightarrow \infty} z^{-2 \pi-1} f(z)<+\infty .
\end{aligned}
$$

Remark that the point $\infty$ is a generalized zero of nonpositive type of multiplicity $\pi$ of $f(z) \in \mathcal{N}_{\kappa}$ if and only if 0 is a generalized zero of nonpositive type of multiplicity $\pi$ of $f\left(-z^{-1}\right) \in \mathcal{N}_{\kappa}$ and that the point $\infty$ is a generalized pole of nonpositive type of multiplicity $\pi$ of $f(z) \in \mathcal{N}_{\kappa}$ if and only if the point $\infty$ is a generalized zero of nonpositive type of multiplicity $\pi$ of $-f(z)^{-1} \in \mathcal{N}_{\kappa}$ (see [8] for the proof of sufficiency).

In 1981, Krein and Langer [7] showed that the total multiplicity of poles (zeros, respectively) in $\mathbb{C}^{+}$and generalized poles (generalized zeros, respectively) of nonpositive type in $\mathbb{R} \cup\{\infty\}$ of $f(z) \in \mathcal{N}_{\kappa}$ is equal to $\kappa$, which implies that each $f(z) \in \mathcal{N}_{\kappa}$ has finite number of zeros and poles in $\mathbb{C}^{+}$. As a corollary of this result, we infer ([9, Proposition 3.2]) that if $f(z) \in \mathcal{N}_{\kappa_{1}}$ and $g(z) \in \mathcal{N}_{\kappa_{2}}$ have no common poles in $\mathbb{C}^{+}$and common generalized poles of nonpositive type in $\mathbb{R} \cup\{\infty\}$, then $f(z)+g(z) \in \mathcal{N}_{\kappa_{1}+\kappa_{2}}$.

By means of an operator representation of $f(z) \in \mathcal{N}_{\kappa}$, in 1985, Daho and Langer [9, Proposition 2.1] derived an integral representation of $f(z) \in \mathcal{N}_{\kappa}$ as follows:

$$
\begin{aligned}
f(z)= & \frac{1}{\prod_{j=1}^{\kappa_{1}}\left(z-\alpha_{j}\right)\left(z-\bar{\alpha}_{j}\right)} \\
& \times\left(\left(z^{2}+y_{0}^{2}\right)^{\kappa} \int_{\mathbb{R}} \frac{u z+y_{0}^{2}}{u-z} \mathrm{~d} \sigma(u)+\sum_{i=0}^{2 \kappa+1} b_{i} z^{i}\right),
\end{aligned}
$$

where $\sigma(u)$ is a positive measure on $\mathbb{R}$ satisfying $\int_{\mathbb{R}} \mathrm{d} \sigma(u)<$ $+\infty, \alpha_{1}, \ldots, \alpha_{\kappa_{1}}$ are either the poles of $f(z)$ in $\mathbb{C}^{+}$or its generalized poles of nonpositive type on $\mathbb{R}$, counted according to their multiplicities, $b_{0}, b_{1}, \ldots, b_{2 \kappa+1} \in \mathbb{R}$ with $b_{2 \kappa+1} \geq 0$, and $y_{0}$ is a given positive number such that $i y_{0} \in D(f)$.

In 2000, Dijksma et al. [6] gave an interesting factorization for each function $f(z) \in \mathcal{N}_{\kappa}$. More precisely, if $\alpha_{1}, \ldots, \alpha_{\kappa_{1}}$ are all the zeros in $\mathbb{C}^{+}$and the generalized zeros of nonpositive type of $f(z) \in \mathcal{N}_{\kappa}$ on $\mathbb{R}$ and $\beta_{1}, \ldots, \beta_{\kappa_{2}}$ are all the poles in $\mathbb{C}^{+}$and the generalized poles of nonpositive type of $f(z) \in \mathcal{N}_{\kappa}$ on $\mathbb{R}$, all counted according to their multiplicities, then

$$
f(z)=\frac{\prod_{i=1}^{\kappa_{1}}\left(z-\alpha_{i}\right)\left(z-\bar{\alpha}_{i}\right)}{\prod_{i=1}^{\kappa_{2}}\left(z-\beta_{i}\right)\left(z-\bar{\beta}_{i}\right)} f_{0}(z),
$$

where $f_{0}(z) \in \mathcal{N}_{0}$. The factorization result (11) of $f(z) \in \mathcal{N}_{\kappa}$, together with the integral representation (3) of $f_{0}(z) \in \mathcal{N}_{0}$, gives rise to the following result. Each $f(z) \in \mathcal{N}_{\mathcal{K}}$ admits an integral representation

$$
\begin{aligned}
f(z)= & \frac{\prod_{i=1}^{\kappa_{1}}\left(z-\alpha_{i}\right)\left(z-\bar{\alpha}_{i}\right)}{\prod_{i=1}^{\kappa_{2}}\left(z-\beta_{i}\right)\left(z-\bar{\beta}_{i}\right)} \\
& \times\left(\alpha z+\beta+\int_{\mathbb{R}}\left(\frac{1}{u-z}-\frac{u}{1+u^{2}}\right) \mathrm{d} \sigma(u)\right),
\end{aligned}
$$

where $\alpha_{i}, \beta_{i}, \kappa_{1}, \kappa_{2}$ are the same as in (11), and $\alpha \geq 0$, $\beta \in \mathbb{R}$, and $\sigma(u)$ is a positive measure on $\mathbb{R}$ satisfying $\int_{\mathbb{R}}\left(1+u^{2}\right)^{-1} \mathrm{~d} \sigma(u)<+\infty$.

It should be noted that generalized Nevanlinna functions are closely related to the theory of extensions of symmetric operators in the Pontryagin spaces. The reader can refer to $[1,7,10-13]$ and the references therein.

In this paper, we present some interesting propositions on generalized Nevanlinna functions in the class $\mathcal{N}_{\kappa}$. Firstly, we show that the number of negative eigenvalues of each Hermitian generalized Loewner matrix generated by a function $f(z) \in \mathcal{N}_{\kappa}$ is not greater than $\kappa$. This leads to an equivalent definition of the generalized Nevanlinna function with negative index $\kappa$ (see Theorem 7 below), which extends the original one to the multiple point case. Secondly, we indicate that the number of negative eigenvalues of the 
Hankel matrix, constructed with partial coefficients of the asymptotic expansion of a function $f(z) \in \mathcal{N}_{\kappa}$ at a real point or at infinity, does not exceed $\kappa$ (see Theorems 14 and 15). Finally, we give an explicit formula of the negative index of real rational functions by using the relations among Loewner, Bézout, and Hankel matrices (see Theorem 21). These results will provide first tools for the solution of the indefinite truncated moment problems together with the multiple Nevanlinna-Pick interpolation problems in the class $\mathcal{N}_{\kappa}$, based on the so-called Hankel vector approach, in subsequent work.

\section{An Equivalent Definition of Generalized Nevanlinna Functions of the Class $\mathcal{N}_{\kappa}$}

In this section, we will show that the Hermitian Loewner matrix $P_{f}\left(z_{1}, \ldots, z_{m}\right)$ appearing in the definition of generalized Nevanlinna functions with negative index $\kappa$ can be substituted by a certain Hermitian generalized Loewner matrix. For a more general form of generalized Loewner matrices, the reader can refer to $[14,15]$.

For convenience, we introduce the notion of the divided difference of a complex function of one complex variable. Let $f(z)$ be such a function of one complex variable $z$, let $\left(z_{i}\right)_{i \in \mathbb{N}^{+}}$ be a sequence of distinct points located in the domain of $f(z)$, and let $\left[z_{i}\right]_{f}:=f\left(z_{i}\right), i=1,2, \ldots$, and, in the case $k \geq 1$, moreover,

$$
\left[z_{1}, \ldots, z_{k+1}\right]_{f}:=\frac{\left[z_{1}, \ldots, z_{k}\right]_{f}-\left[z_{2}, \ldots, z_{k+1}\right]_{f}}{z_{1}-z_{k+1}}
$$

Such $\left[z_{1}, \ldots, z_{k+1}\right]_{f}$ is called the $k$ th divided difference of $f(z)$ at $z_{1}, \ldots, z_{k+1}$.

In the sequel, we give some properties of the higher divided difference of the complex functions in one complex variable. These properties are very similar to those of the higher divided difference of the real functions of one real variable. But the proofs of them need to be checked carefully.

Proposition 1. Let $D$ be an open disk in the complex plane, and let $z_{1}, \ldots, z_{n} \in D$ be distinct points. If $f(z)$ is holomorphic in the closed disk $\bar{D}$, then

$$
\left[z_{\pi(1)}, \ldots, z_{\pi(n)}\right]_{f}=\left[z_{1}, \ldots, z_{n}\right]_{f}
$$

in which $\pi$ is an arbitrary $n$-permutation.

Proof. By using Cauchy's Integral Formula, we infer that

$$
f\left(z_{j}\right)=\frac{1}{2 \pi i} \int_{\partial D} \frac{f(\xi)}{\xi-z_{j}} \mathrm{~d} \xi, \quad j=1, \ldots, n .
$$

Then, for each $n$-permutation $\pi$, we have

$$
\begin{aligned}
& {\left[z_{\pi(1)}, z_{\pi(2)}\right]_{f}=\frac{1}{2 \pi i} \int_{\partial D} \frac{f(\xi)}{\left(\xi-z_{\pi(1)}\right)\left(\xi-z_{\pi(2)}\right)} \mathrm{d} \xi,} \\
& {\left[z_{\pi(1)}, z_{\pi(2)}, z_{\pi(3)}\right]_{f}} \\
& =\frac{1}{2 \pi i} \int_{\partial D} \frac{f(\xi)}{\left(\xi-z_{\pi(1)}\right)\left(\xi-z_{\pi(2)}\right)\left(\xi-z_{\pi(3)}\right)} \mathrm{d} \xi, \\
& {\left[z_{\pi(1)}, \ldots, z_{\pi(n)}\right]_{f}=\frac{\left[z_{\pi(1)}, \ldots, z_{\pi(n-1)}\right]_{f}-\left[z_{\pi(2)}, \ldots, z_{\pi(n)}\right]_{f}}{z_{\pi(1)}-z_{\pi(n)}}} \\
& =\frac{1}{z_{\pi(1)}-z_{\pi(n)}} \\
& \times\left(\frac{1}{2 \pi i} \int_{\partial D} \frac{f(\xi) \mathrm{d} \xi}{\left(\xi-z_{\pi(1)}\right) \cdots\left(\xi-z_{\pi(n-1)}\right)}\right. \\
& \left.-\frac{1}{2 \pi i} \int_{\partial D} \frac{f(\xi) \mathrm{d} \xi}{\left(\xi-z_{\pi(2)}\right) \cdots\left(\xi-z_{\pi(n)}\right)}\right) \\
& =\frac{1}{2 \pi i} \int_{\partial D} \frac{f(\xi)}{\left(\xi-z_{\pi(1)}\right) \cdots\left(\xi-z_{\pi(n)}\right)} \mathrm{d} \xi \\
& =\frac{1}{2 \pi i} \int_{\partial D} \frac{f(\xi)}{\left(\xi-z_{1}\right) \cdots\left(\xi-z_{n}\right)} \mathrm{d} \xi \text {. }
\end{aligned}
$$

The last equation implies that (14) holds for each $n$ permutation $\pi$.

With the proof of Proposition 1, we can prove the following two statements.

Proposition 2. If $f(z)$ is holomorphic at the point $\alpha \in \mathbb{C}$ and $z_{1}, z_{2}, \ldots, z_{n+1}$ are distinct and variable points in $\mathbb{C}$, then

$$
\lim _{z_{i} \rightarrow \alpha, \forall i}\left[z_{1}, \ldots, z_{n+1}\right]_{f}=\frac{1}{n !} f^{(n)}(\alpha)=:[\underbrace{\alpha, \ldots, \alpha}_{n+1}]_{f}
$$

holds for an arbitrary $n \in \mathbb{N}^{+}$.

Proof. Since $f(z)$ is holomorphic at $\alpha$, there exists $\delta>0$ such that $f(z)$ is holomorphic in $\bar{D}:=\{z \in \mathbb{C}|| z-\alpha \mid \leq \delta\}$. It follows from the proof of Proposition 1 that

$$
\begin{aligned}
\lim _{z_{i} \rightarrow \alpha, \forall i} & {\left[z_{1}, \ldots, z_{n+1}\right]_{f} } \\
= & \lim _{z_{i} \rightarrow \alpha, \forall i} \frac{1}{2 \pi i} \int_{\partial D} \frac{f(\xi)}{\left(\xi-z_{1}\right) \cdots\left(\xi-z_{n+1}\right)} \mathrm{d} \xi \\
= & \frac{1}{2 \pi i} \int_{\partial D} \frac{f(\xi)}{(\xi-\alpha)^{n+1}} \mathrm{~d} \xi \\
= & \frac{1}{n !} f^{(n)}(\alpha), \quad \forall n \in \mathbb{N}^{+} .
\end{aligned}
$$

Then (17) follows. 
Proposition 3. If $f(z)$ is holomorphic at two distinct points $\alpha, \beta \in \mathbb{C}$ and $z_{1}, \ldots, z_{m+1}, w_{1}, \ldots, w_{n+1}$ are distinct and variable points in $\mathbb{C}$, then

$$
\begin{aligned}
& \lim _{\substack{z_{j} \rightarrow \alpha, \forall j \\
w_{k} \rightarrow \beta, \forall k}}\left[z_{1}, \ldots, z_{m+1}, w_{1}, \ldots, w_{n+1}\right]_{f} \\
& \quad=\left.\frac{1}{m ! n !} \frac{\partial^{m+n}}{\partial z^{m} \partial w^{n}}\left(\frac{f(z)-f(w)}{z-w}\right)\right|_{z=\alpha, w=\beta} \\
& =:[\underbrace{\alpha, \ldots, \alpha}_{m+1}, \underbrace{\beta, \ldots, \beta}_{n+1}]_{f}
\end{aligned}
$$

holds for any $m, n \in \mathbb{N}^{+}$.

Proof. Since $f(z)$ is holomorphic at the points $\alpha$ and $\beta$, there exist two disks $D_{1}$ and $D_{2}$ with centers at $\alpha$ and $\beta$, respectively, such that $\bar{D}_{1} \cap \bar{D}_{2}=\varnothing$ and $f(z)$ is holomorphic in $\bar{D}_{1}$ and $\bar{D}_{2}$. By using Cauchy's Integral Formula,

$$
\begin{aligned}
& f\left(z_{j}\right)=\frac{1}{2 \pi i} \int_{\partial D_{1} \cup \partial D_{2}} \frac{f(\xi)}{\xi-z_{j}} \mathrm{~d} \xi \\
& f\left(w_{k}\right)=\frac{1}{2 \pi i} \int_{\partial D_{1} \cup \partial D_{2}} \frac{f(\xi)}{\xi-w_{k}} \mathrm{~d} \xi,
\end{aligned}
$$

where $z_{j} \in D_{1}, w_{k} \in D_{2}(j=1, \ldots, m+1, k=1, \ldots, n+1)$. Therefore,

$$
\begin{aligned}
& \lim _{\substack{z_{j} \rightarrow \alpha, \forall j \\
w_{k} \rightarrow \beta, \forall k}}\left[z_{1}, \ldots, z_{m+1}, w_{1}, \ldots, w_{n+1}\right]_{f} \\
& =\lim _{\substack{z_{j} \rightarrow \alpha, \forall j \\
w_{k} \rightarrow \beta, \forall k}} \frac{1}{2 \pi i}
\end{aligned}
$$

$$
\begin{aligned}
& \times \int_{\partial D_{1} \cup \partial D_{2}} \frac{f(\xi)}{\left(\xi-z_{1}\right) \cdots\left(\xi-z_{m+1}\right)\left(\xi-w_{1}\right) \cdots\left(\xi-w_{n+1}\right)} \mathrm{d} \xi \\
= & \frac{1}{2 \pi i} \int_{\partial D_{1} \cup \partial D_{2}} \frac{f(\xi)}{(\xi-\alpha)^{m+1}(\xi-\beta)^{n+1}} \mathrm{~d} \xi .
\end{aligned}
$$

On the other hand,

$$
\begin{aligned}
& \left.\frac{1}{m ! n !} \frac{\partial^{m+n}}{\partial z^{m} \partial w^{n}}\left(\frac{f(z)-f(w)}{z-w}\right)\right|_{z=\alpha, w=\beta} \\
& =\left.\frac{1}{m ! n !} \frac{\partial^{m+n}}{\partial z^{m} \partial w^{n}}\left(\frac{1}{2 \pi i} \int_{\partial D_{1} \cup \partial D_{2}} \frac{f(\xi) \mathrm{d} \xi}{(\xi-z)(\xi-w)}\right)\right|_{z=\alpha, w=\beta} \\
& =\frac{1}{2 \pi i} \int_{\partial D_{1} \cup \partial D_{2}} \frac{f(\xi) \mathrm{d} \xi}{(\xi-\alpha)^{m+1}(\xi-\beta)^{n+1}} .
\end{aligned}
$$

Combining (21) and (22), we obtain (19) immediately.

The following lemma plays an important role by proving the fact that the number of negative eigenvalues of the Hermitian generalized Loewner matrices generated by a function $f(z) \in \mathscr{N}_{\kappa}$ does not exceed $\kappa$.

Lemma 4. Let $f(z)$ be a complex function of one complex variable, and let $z_{1}, \ldots, z_{m}, w_{1}, \ldots, w_{n}$ be distinct points in the domain of $f(z)$. Then

$$
M\left(\left[z_{i}, w_{j}\right]_{f}\right)_{i, j=1}^{m, n} N^{\mathrm{T}}=\left(\left[z_{1}, \ldots, z_{i}, w_{1}, \ldots, w_{j}\right]_{f}\right)_{i, j=1}^{m, n},
$$

in which $M:=M_{m-1} \cdots M_{2} M_{1}$ and $N:=N_{n-1} \cdots N_{2} N_{1}$ with

$$
\begin{aligned}
& M_{k}:=\left(\begin{array}{ccccc}
I_{k-1} & & & & \\
& 1 & & & \\
& -\left(z_{k+1}-z_{1}\right)^{-1} & \left(z_{k+1}-z_{1}\right)^{-1} & & \\
& & \ddots & \ddots & \\
& & & -\left(z_{m}-z_{m-k}\right)^{-1} & \left(z_{m}-z_{m-k}\right)^{-1}
\end{array}\right),
\end{aligned}
$$

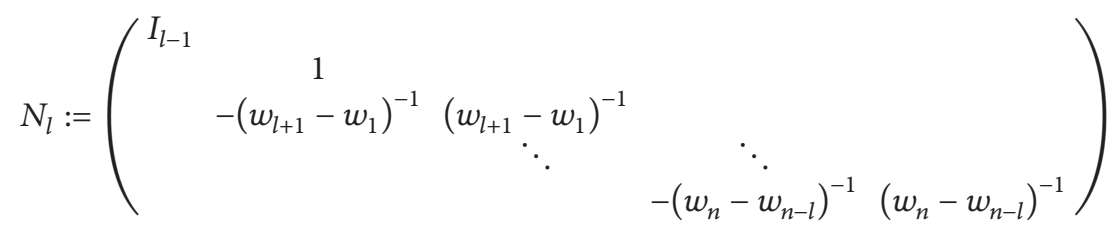

being nonsingular matrices for $k=1, \ldots, m-1$ and $l=$ $1, \ldots, n-1$.
Proof. Let $P_{k}:=\left(\begin{array}{c}A_{k} \\ B_{k}\end{array}\right)$ and $Q_{l}:=\left(C_{l}, D_{l}\right)$, in which, for $k=$ $0,1, \ldots, m-1$, 


$$
\begin{gathered}
A_{k}:=\left(\begin{array}{ccc}
{\left[z_{1}, w_{1}\right]_{f}} & \ldots & {\left[z_{1}, w_{n}\right]_{f}} \\
\vdots & & \vdots \\
{\left[z_{1}, \ldots, z_{k+1}, w_{1}\right]_{f}} & \ldots & {\left[z_{1}, \ldots, z_{k+1}, w_{n}\right]_{f}}
\end{array}\right), \\
B_{k}:=\left(\begin{array}{ccc}
{\left[z_{2}, \ldots, z_{k+2}, w_{1}\right]_{f}} & \cdots & {\left[z_{2}, \ldots, z_{k+2}, w_{n}\right]_{f}} \\
\vdots & & \vdots \\
{\left[z_{m-k}, \ldots, z_{m}, w_{1}\right]_{f}} & \cdots & {\left[z_{m-k}, \ldots, z_{m}, w_{n}\right]_{f}}
\end{array}\right),
\end{gathered}
$$

and, for $l=0,1, \ldots, n-1$,

$$
\begin{gathered}
C_{l}:=\left(\begin{array}{ccc}
{\left[z_{1}, w_{1}\right]_{f}} & \ldots & {\left[z_{1}, w_{1}, \ldots, w_{l+1}\right]_{f}} \\
\vdots & & \vdots \\
{\left[z_{1}, \ldots, z_{m}, w_{1}\right]_{f}} & \cdots & {\left[z_{1}, \ldots, z_{m}, w_{1}, \ldots, w_{l+1}\right]_{f}}
\end{array}\right), \\
D_{l}:=\left(\begin{array}{ccc}
{\left[z_{1}, w_{2}, \ldots, w_{l+2}\right]_{f}} & \cdots & {\left[z_{1}, w_{n-l}, \ldots, w_{n}\right]_{f}} \\
\vdots & & \vdots \\
{\left[z_{1}, \ldots, z_{m}, w_{2}, \ldots, w_{l+2}\right]_{f}} & \cdots & {\left[z_{1}, \ldots, z_{m}, w_{n-l}, \ldots, w_{n}\right]_{f}}
\end{array}\right) .
\end{gathered}
$$

Obviously, $P_{0}=\left(\left[z_{i}, w_{j}\right]_{f}\right)_{i, j=1}^{m, n}, Q_{0}=P_{m-1}$, and $Q_{n-1}=$ $\left(\left[z_{1}, \ldots, z_{i}, w_{1}, \ldots, w_{j}\right]_{f}\right)_{i, j=1}^{m, n}$. By Proposition 1 , we check easily that

$$
M_{k}\left(\begin{array}{c}
A_{k-1} \\
B_{k-1}
\end{array}\right)=\left(\begin{array}{c}
A_{k} \\
B_{k}
\end{array}\right), \quad\left(C_{l-1}, D_{l-1}\right) N_{l}^{\mathrm{T}}=\left(C_{l}, D_{l}\right) ;
$$

that is, $M_{k} P_{k-1}=P_{k}$ and $Q_{l-1} N_{l}^{\mathrm{T}}=Q_{l} \quad(k=1, \ldots, m-1, l=$ $1, \ldots, n-1)$. Hence we have

$$
M P_{0} N^{\mathrm{T}}=M_{m-1} \cdots M_{2} M_{1} P_{0} N_{1}^{\mathrm{T}} N_{2}^{\mathrm{T}} \cdots N_{n-1}^{\mathrm{T}}=Q_{n-1},
$$

as asserted.

In particular, if, in Lemma $4, f(z) \in \mathcal{N}_{\kappa}, m=n$, and $w_{i}=\bar{z}_{i} \quad(i=1, \ldots, m)$, then $N^{\mathrm{T}}=M^{*}$ and thus (23) in this case can be rewritten as follows:

$$
M\left(\left[z_{i}, \bar{z}_{j}\right]_{f}\right)_{i, j=1}^{m} M^{*}=\left(\left[z_{1}, \ldots, z_{i}, \bar{z}_{1}, \ldots, \bar{z}_{j}\right]_{f}\right)_{i, j=1}^{m} .
$$

Suppose now that $f(z) \in \mathcal{N}_{\kappa}$ and $z_{1}, \ldots, z_{\rho} \in \mathbb{C}^{+} \cap$ $D(f)$ are mutually different, with multiplicities $\tau_{1}, \ldots, \tau_{\rho}$, respectively. For each $z_{i}$, there exist mutually different points $z_{i j}^{(k)} \in \mathbb{C}^{+} \cap D(f)\left(j=1, \ldots, \tau_{i}, k=1,2, \ldots\right)$ such that

$$
\lim _{k \rightarrow+\infty} z_{i j}^{(k)}=z_{i}, \quad i=1, \ldots, \rho, j=1, \ldots, \tau_{i} .
$$

Therefore, Proposition 3 says

$$
\begin{gathered}
\lim _{k \rightarrow+\infty}\left[z_{i 1}^{(k)}, \ldots, z_{i u}^{(k)}, \overline{z_{j 1}^{(k)}}, \ldots, \overline{z_{j \nu}^{(k)}}\right]_{f} \\
=[\underbrace{z_{i}, \ldots, z_{i}}_{u}, \underbrace{\bar{z}_{j}, \ldots, \bar{z}_{j}}_{\nu}]_{f},
\end{gathered}
$$

in which $i, j=1, \ldots, \rho, u=1, \ldots, \tau_{i}$, and $v=1, \ldots, \tau_{j}$. Since $f(z) \in \mathcal{N}_{\kappa}$ and all the $z_{i j}^{(k)} \in \mathbb{C}^{+} \cap D(f)$ are distinct points, we have

$$
\nu\left(P_{f}\left(z_{11}^{(k)}, \ldots, z_{1 \tau_{1}}^{(k)}, \ldots, z_{\rho 1}^{(k)}, \ldots, z_{\rho \tau_{p}}^{(k)}\right)\right) \leq \kappa, \quad k=1,2, \ldots
$$

Observe that

$$
P_{f}\left(z_{11}^{(k)}, \ldots, z_{1 \tau_{1}}^{(k)}, \ldots, z_{\rho 1}^{(k)}, \ldots, z_{\rho \tau_{\rho}}^{(k)}\right)=\left(P_{i j}^{(k)}\right)_{i, j=1}^{\rho},
$$

in which

$$
P_{i j}^{(k)}:=\left(\left[z_{i u}^{(k)}, \overline{z_{j \nu}^{(k)}}\right]_{f}\right)_{u, v=1}^{\tau_{i}, \tau_{j}}, \quad i, j=1, \ldots, \rho .
$$

By use of (23), there exist nonsingular matrices $T_{i}^{(k)} \quad(i=$ $1, \ldots, \rho)$ such that

$$
\begin{aligned}
\widetilde{P}_{i j}^{(k)} & :=T_{i}^{(k)} P_{i j}^{(k)} T_{j}^{(k)^{*}} \\
& =\left(\left[z_{i 1}^{(k)}, \ldots, z_{i u}^{(k)}, \overline{z_{j 1}^{(k)}}, \ldots, \overline{z_{j \nu}^{(k)}}\right]_{f}\right)_{u, v=1}^{\tau_{i}, \tau_{j}}, \quad i, j=1, \ldots, \rho,
\end{aligned}
$$

or equivalently,

$$
\begin{aligned}
\widetilde{P}_{f}^{(k)}:= & \left(\widetilde{P}_{i j}^{(k)}\right)_{i, j=1}^{\rho} \\
= & \operatorname{diag}\left(T_{1}^{(k)}, \ldots, T_{\rho}^{(k)}\right) \\
& \quad \times P_{f}\left(z_{11}^{(k)}, \ldots, z_{1 \tau_{1}}^{(k)}, \ldots, z_{\rho 1}^{(k)}, \ldots, z_{\rho \tau_{\rho}}^{(k)}\right) \\
& \quad \times \operatorname{diag}\left(T_{1}^{(k)}, \ldots, T_{\rho}^{(k)}\right)^{*} .
\end{aligned}
$$

The last equation together with (32) implies that

$$
\begin{aligned}
\nu\left(\widetilde{P}_{f}^{(k)}\right) & =v\left(P_{f}\left(z_{11}^{(k)}, \ldots, z_{1 \tau_{1}}^{(k)}, \ldots, z_{\rho 1}^{(k)}, \ldots, z_{\rho \tau_{\rho}}^{(k)}\right)\right) \\
& \leq \kappa, \quad k=1,2, \ldots
\end{aligned}
$$

Let $f(z) \in \mathcal{N}_{\kappa}$ and let $z_{1}, \ldots, z_{\rho} \in \mathbb{C}^{+} \cap D(f)$ be distinct points with multiplicities $\tau_{1}, \ldots, \tau_{\rho}$, respectively. Define the generalized Loewner matrix generated by the function $f(z) \epsilon$ $\mathcal{N}_{\kappa}$ and such $z_{1}, \ldots, z_{\rho}$ by

$$
\begin{aligned}
& P_{f}(\underbrace{z_{1}, \ldots, z_{1}}_{\tau_{1}}, \ldots, \underbrace{z_{\rho}, \ldots, z_{\rho}}_{\tau_{\rho}}):=\left(P_{i j}\right)_{i, j=1}^{\rho}, \\
P_{i j}:= & \left([\underbrace{z_{i}, \ldots, z_{i}}_{u}, \underbrace{\bar{z}_{j}, \ldots, \bar{z}_{j}}_{\nu}]_{f}\right)_{u, v=1}^{\tau_{i}, \tau_{j}}, \quad i, j=1, \ldots, \rho .
\end{aligned}
$$

Proposition 3 indicates that

$$
\lim _{k \rightarrow+\infty} \widetilde{P}_{f}^{(k)}=P_{f}(\underbrace{z_{1}, \ldots, z_{1}}_{\tau_{1}}, \ldots, \underbrace{z_{\rho}, \ldots, z_{\rho}}_{\tau_{\rho}})=\left(P_{i j}\right)_{i, j=1}^{\rho},
$$

where $\widetilde{P}_{f}^{(k)}$ is defined by (36).

Thus, from (2) and the fact that each eigenvalue of a square matrix is a continuous function of its entries, we immediately obtain the following result. 
Theorem 5. Let $f(z) \in \mathcal{N}_{\kappa}$, and let $z_{1}, \ldots, z_{\rho} \in \mathbb{C}^{+} \cap D(f)$ be distinct points with multiplicities $\tau_{1}, \ldots, \tau_{\rho}$ (each $\left.\tau_{i} \geq 1\right)$, respectively. Then the Hermitian generalized Loewner matrix

$$
P_{f}(\underbrace{z_{1}, \ldots, z_{1}}_{\tau_{1}}, \ldots, \underbrace{z_{\rho}, \ldots, z_{\rho}}_{\tau_{\rho}})
$$

defined by (38) has at most $\kappa$ negative eigenvalues.

Proof. Since $f(z) \in \mathcal{N}_{\kappa}$, by (2), we have $\nu\left(\widetilde{P}_{f}^{(k)}\right) \leq \kappa$ for $k=$ $1,2, \ldots$. It follows from (39) and the continuity of eigenvalues of a square matrix that

$$
\nu\left(P_{f}(\underbrace{z_{1}, \ldots, z_{1}}_{\tau_{1}}, \ldots, \underbrace{z_{\rho}, \ldots, z_{\rho}}_{\tau_{\rho}})\right) \leq \kappa .
$$

Thus we complete the proof.

Observe that, by Proposition 3, each entry of $P_{i j}$ defined by (38) can be rewritten as follows:

$$
\begin{aligned}
& {[\underbrace{z_{i}, \ldots, z_{i}}_{k+1}, \underbrace{\bar{z}_{j}, \ldots, \bar{z}_{j}}_{l+1}]_{f}} \\
& =\left.\frac{1}{k ! l !} \frac{\partial^{k+l}}{\partial z^{k} \partial w^{l}}\left(\frac{f(z)-f(w)}{z-w}\right)\right|_{z=z_{i}, w=\bar{z}_{j}}, \quad \forall i, j, k, l .
\end{aligned}
$$

Then the Loewner matrix appearing in the definition of generalized Nevanlinna functions of the class $\mathcal{N}_{\mathcal{K}}$ is a special case of the generalized Loewner matrix $\left(P_{i j}\right)_{i, j=1}^{\rho}$ defined by (38).

Theorem 6. Let $f(z)$ be meromorphic in $\mathbb{C} \backslash \mathbb{R}$ satisfying $f(\bar{z})=\overline{f(z)}$ for $z \in D(f)$. If

$$
\begin{aligned}
& \max \{ \nu\left(P_{f}(\underbrace{z_{1}, \ldots, z_{1}}_{\tau_{1}}, \ldots, \underbrace{z_{\rho}, \ldots, z_{\rho}}_{\tau_{\rho}})\right) \\
& \mid \rho, \tau_{1}, \ldots, \tau_{\rho} \in \mathbb{N}^{+}, \\
&\left.z_{1}, \ldots, z_{\rho} \in \mathbb{C}^{+} \cap D(f) \text { different }\right\}=\kappa,
\end{aligned}
$$

then $f(z) \in \mathcal{N}_{\kappa}$.

Proof. It follows from (43) that, for an arbitrary $m \in \mathbb{N}^{+}$ and for any distinct points $z_{1}^{\prime}, \ldots, z_{m}^{\prime} \in \mathbb{C}^{+} \cap D(f)$, $\nu\left(P_{f}\left(z_{1}^{\prime}, \ldots, z_{m}^{\prime}\right)\right) \leq \kappa$, and, for some choice of $\rho, \tau_{1}, \ldots, \tau_{\rho}$ and distinct points $z_{1}, \ldots, z_{\rho} \in \mathbb{C}^{+} \cap D(f)$,

$$
\nu\left(P_{f}(\underbrace{z_{1}, \ldots, z_{1}}_{\tau_{1}}, \ldots, \underbrace{z_{\rho}, \ldots, z_{\rho}}_{\tau_{\rho}})\right)=\kappa .
$$

Let now $m:=\tau_{1}+\cdots+\tau_{\rho} \in \mathbb{N}^{+}$and let $D_{z_{i}, \varepsilon}:=\{z \in$ $\left.\mathbb{C}|| z-z_{i} \mid<\varepsilon\right\}, \forall \varepsilon>0$. Taking $\varepsilon>0$ small enough such that $D_{z_{i}, \varepsilon} \cap D_{z_{j}, \varepsilon}=\emptyset, \forall i \neq j$, we can choose different points $z_{i 1}, \ldots, z_{i \tau_{i}}$ in $D_{z_{i}, \varepsilon} \cap \mathbb{C}^{+} \cap D(f)$ for $i=1, \ldots, \rho$ instead of the original multiple point $z_{i}(i=1, \ldots, \rho)$ such that

$$
\nu\left(P_{f}\left(z_{11}, \ldots, z_{1 \tau_{1}}, \ldots, z_{\rho 1}, \ldots, z_{\rho \tau_{\rho}}\right)\right)=\kappa .
$$

This is possible, because of (36) and (39) together with the continuity of the eigenvalues of a square matrix for its entries. By definition, we obtain from the afore-stated results that $f(z) \in \mathcal{N}_{\kappa}$. Thus the proof is complete.

In view of Theorems 5 and 6 , we present now the announced result, that is, an equivalent definition of generalized Nevanlinna functions of the class $\mathcal{N}_{\kappa}$ in some sense, which extends the original one to the multiple point case.

Theorem 7. Let $f(z)$ be a meromorphic function in $\mathbb{C} \backslash \mathbb{R}$ such that $f(\bar{z})=\overline{f(z)}$ for $z \in D(f)$. Then $f(z)$ is a generalized Nevanlinna function of the class $\mathcal{N}_{\kappa}$, if and only if (41) holds for any $\rho \in \mathbb{N}^{+}$and any distinct points $z_{1}, \ldots, z_{\rho} \in \mathbb{C}^{+} \cap D(f)$ with multiplicities $\tau_{1}, \ldots, \tau_{\rho}$ (each $\tau_{i} \geq 1$ ), respectively, and, for at least one such choice, the equality in (41) holds.

\section{Some Propositions on Asymptotic Expansions of $f(z)$ of the Class $\mathcal{N}_{\kappa}$}

In this section, we study some propositions on asymptotic expansions of a function $f(z) \in \mathcal{N}_{\kappa}$ at $t \in \mathbb{R}$ and at infinity. To begin with, we introduce the notion of angular derivative of a meromorphic function $f(z)$ defined on $\mathbb{C}^{+}$at a real point $t$.

Let $f(z)$ be meromorphic in $\mathbb{C}^{+}$and let $t \in \mathbb{R}$. If the nontangential limits

$$
\begin{gathered}
f(t):=\angle \lim _{z \rightarrow t}[z]_{f}, \\
f^{\prime}(t):=\angle \lim _{z \rightarrow t}[z, t]_{f}=\angle \lim _{z \rightarrow t} \frac{f(z)-f(t)}{z-t}
\end{gathered}
$$

exist, then we say that $f(z)$ has an angular derivative (of order 1 ) at $t$, denoted by $f^{\prime}(t)$. Sometimes we write $f(t)$ and $f^{\prime}(t)$ as $[t]_{f}$ and $[t, t]_{f}$, respectively.

More generally, for an arbitrary $k \in \mathbb{N}^{+}$, we say that $f(z)$ has the $k$ th angular derivative at a real point $t$, denoted by $f^{(k)}(t)$, if the nontangential limits

$$
\begin{aligned}
& {[\underbrace{t, \ldots, t}_{j+1}]_{f}} \\
& =\angle \lim _{z \rightarrow t}[z, \underbrace{t, \ldots, t}_{j}]_{f} \\
& =\angle \lim _{z \rightarrow t} \frac{[z, \overbrace{t, \ldots, t}^{j-1}]_{f}-[\overbrace{t, \ldots, t}^{j}]_{f}}{z-t} \quad(j=0,1, \ldots, k)
\end{aligned}
$$

exist, and in this case $f^{(k)}(t):=k ![\underbrace{t, \ldots, t}_{k+1}]_{f}, \forall k \geq 0$. 
In [16], Chen and Hu proved an interesting property on higher angular derivatives of a function $f(z)$ in the class $\mathcal{N}_{0}$ at a real point.

Lemma 8. Let $t \in \mathbb{R}$ and let $f(z) \in \mathcal{N}_{0}$ have an integral representation (3), in which $\alpha \geq 0, \beta \in \mathbb{R}$, and $\sigma(u)$ is a positive measure on $\mathbb{R}$ such that (4) holds. Then, for some $m \in$ $\mathbb{N}^{+}$, the angular derivatives $f^{(k)}(t) \quad(k=0,1, \ldots, 2 m-1)$ exist and are real numbers if and only if $\int_{\mathbb{R}}(u-t)^{-2 m} \mathrm{~d} \sigma(\mathrm{u})<+\infty$. In that case,

$$
\begin{aligned}
& f^{(k)}(t)
\end{aligned}
$$

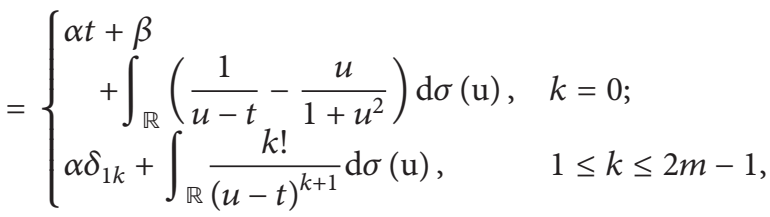

in which $\delta_{i j}$ stands for Kronecker symbol, that is, when $i=j$, $\delta_{i j}=1$; when $i \neq j, \delta_{i j}=0$.

It follows from Lemma 8 that if $f^{(k)}(t)(k=0,1, \ldots, 2 m-$ 1) exist and are real numbers then, for $z \in \mathbb{C}^{+}$,

$$
\begin{aligned}
& \left|f^{(k)}(z)-f^{(k)}(t)\right| \\
& \leq k !\left|\int_{\mathbb{R}}\left(\frac{1}{(u-z)^{k+1}}-\frac{1}{(u-t)^{k+1}}\right) \mathrm{d} \sigma(u)\right| \\
& \leq k !\left|\int_{|u-t|>1}\left(\frac{1}{(u-z)^{k+1}}-\frac{1}{(u-t)^{k+1}}\right) \mathrm{d} \sigma(u)\right| \\
& \quad+k !\left|\int_{|u-t| \leq 1} \frac{(z-t) \sum_{i=0}^{k}(u-z)^{k-i}(u-t)^{i}}{(u-z)^{k+1}(u-t)^{k+1}} \mathrm{~d} \sigma(u)\right| .
\end{aligned}
$$

Obviously, the first term on the right hand side of the inequality (49) tends to 0 when $z$ tends nontangentially to $t$. As for the second term therein, noting $|u-z| \geq \sin \varepsilon|z-t|$ for all $z \in \mathbb{C}^{+}$, in the case of $|u-t| \leq 1$, we have

$$
\begin{aligned}
& \left|\frac{(z-t) \sum_{i=0}^{k}(u-z)^{k-i}(u-t)^{i}}{(u-z)^{k+1}(u-t)^{k+1}}\right| \\
& \leq(u-t)^{-2 m} \sum_{i=0}^{k}\left|\frac{(z-t)(u-t)^{2 m+i-k-1}}{(u-z)^{i+1}}\right| \\
& \quad=(u-t)^{-2 m} \sum_{i=0}^{k}\left|\frac{z-t}{u-z}\right| \cdot\left|\frac{u-t}{u-z}\right|^{i}|u-t|^{2 m-1-k} \\
& \leq(u-t)^{-2 m} \sum_{i=0}^{k}\left|\frac{z-t}{u-z}\right| \cdot\left|\frac{u-t}{u-z}\right|^{i} \\
& =(u-t)^{-2 m} \sum_{i=0}^{k}\left|\frac{z-t}{u-z}\right| \cdot\left|\frac{u-z+z-t}{u-z}\right|^{i}
\end{aligned}
$$

$$
\begin{aligned}
& \leq(u-t)^{-2 m} \sum_{i=0}^{k}\left(1+\left|\frac{z-t}{u-z}\right|\right)^{i+1} \\
& \leq(u-t)^{-2 m} \sum_{i=0}^{k}\left(1+\frac{1}{\sin \varepsilon}\right)^{i+1} .
\end{aligned}
$$

Since $\int_{|u-t| \leq 1}(u-t)^{-2 m} \mathrm{~d} \sigma(u)<+\infty$, by Lebesgue's dominated convergence theorem, the second term on the right hand side of the inequality (49) tends to 0 as well when $z$ tends nontangentially to $t$. Thus

$$
\begin{aligned}
\left|f^{(k)}(z)-f^{(k)}(t)\right| \longrightarrow 0 & \left(z \in \pi_{\varepsilon}(t), z \longrightarrow t\right), \\
& k=0,1, \ldots, 2 m-1 .
\end{aligned}
$$

Therefore, the following statement is valid.

Proposition 9. Let $t \in \mathbb{R}$ and $f(z) \in \mathcal{N}_{0}$. If, for some $m \in \mathbb{N}^{+}$, the angular derivatives $f^{(k)}(t)(k=0,1, \ldots, 2 m-1)$ exist and are real numbers, then

$$
\angle \lim _{z \rightarrow t} f^{(k)}(z)=f^{(k)}(t), \quad k=0,1, \ldots, 2 m-1 .
$$

Next, we verify that each function $f(z)$ in the class $\mathcal{N}_{\kappa}$ has a similar property to that given in Proposition 9. For this purpose, we need the following lemma.

Lemma 10. Let $f(z), g(z)$ be meromorphic in $\mathbb{C}^{+}$, let $t \in \mathbb{R}$, and let $n \in \mathbb{N}^{+}$. If $f(z)$ and $g(z)$ have the angular derivatives of order from 1 to $(n-1)$ at $t$, then

$$
\begin{aligned}
& {[z, \underbrace{t, \ldots, t}_{n}]_{f g}} \\
& =[z, \underbrace{t, \ldots, t}_{n}]_{f}[z]_{g} \\
& \quad+\sum_{i=1}^{n}[\underbrace{t, \ldots, t}_{i}]_{f}[z, \underbrace{t, \ldots, t}_{n+1-i}]_{g}, \quad z \in \mathbb{C}^{+} \cap D(f) \cap D(g) .
\end{aligned}
$$

Proof. In the case of $n=1$, we have

$$
\begin{aligned}
{[z, t]_{f g} } & \\
= & \frac{f(z) g(z)-f(t) g(t)}{z-t}=\frac{f(z)-f(t)}{z-t} g(z) \\
& +f(t) \frac{g(z)-g(t)}{z-t} \\
= & {[z, t]_{f}[z]_{g}+[t]_{f}[z, t]_{g} . }
\end{aligned}
$$

Thus the equality (53) holds for $n=1$. Now we assume that the equality (53) holds for $n=k(k \geq 1)$; that is,

$$
\begin{aligned}
{[z, \underbrace{t, \ldots, t}_{k}]_{f g}=} & {[z, \underbrace{t, \ldots, t}_{k}]_{f}[z]_{g} } \\
& +\sum_{i=1}^{k}[\underbrace{t, \ldots, t}_{i}]_{f}[z, \underbrace{t, \ldots, t}_{k+1-i}]_{g}
\end{aligned}
$$


Then, for the case of $n=k+1$,

$$
\begin{aligned}
{[z, \underbrace{t, \ldots, t}_{k+1}]_{f g} } & =\frac{[z, \overbrace{t, \ldots, t}^{k}]_{f g}-[\overbrace{t, \ldots, t}^{k+1}]_{f g}}{z-t} \\
& =\frac{[z, \overbrace{t, \ldots, t}^{k}}{z z]_{g}-[\overbrace{t, \ldots, t}^{k+1}]_{f}[t]_{g}+\sum_{i=1}^{k}[\overbrace{t, \ldots, t}^{i}]_{f}\left(\left[z, t_{t, \ldots, t}^{k+1-i}\right]_{g}-[\overbrace{t, \ldots, t}^{k+2-i}]_{g}\right)} \\
& =[z, \underbrace{t, \ldots, t}_{k+1}]_{f}[z]_{g}+[\underbrace{t, \ldots, t}_{k+1}]_{f}[z, t]_{g}+\sum_{i=1}^{k}[\underbrace{t, \ldots, t}_{i}]_{f}[z, \underbrace{t, \ldots, t}_{k+2-i}]_{g} \\
& =[z, \underbrace{t, \ldots, t}_{k+1}]_{f}[z]_{g}+\sum_{i=1}^{k+1}[\underbrace{t, \ldots, t}_{i}]_{f}[z, \underbrace{t, \ldots, t}_{k+2-i}]_{g}
\end{aligned}
$$

which means that, for $n=k+1$, the equality (53) is also true. Therefore, by the principle of mathematical induction, we conclude that the equality (53) is valid for all $n \in \mathbb{N}^{+}$.

Let $f(z)$ and $g(z)$ have the angular derivatives of order from 1 to $n$ at $t \in \mathbb{R}$. Then observe that $f(z)+g(z)$ has also the angular derivatives of order from 1 to $n$ at $t$; moreover,

$$
(f+g)^{(k)}(t)=f^{(k)}(t)+g^{(k)}(t), \quad k=0,1, \ldots, n .
$$

On the other hand, by Lemma 10, $f(z) g(z)$ has then the angular derivatives of order from 1 to $n$ at real $t$. As $z$ tends nontangentially to real $t$ in (53), we have

$$
[\underbrace{t, \ldots, t}_{n+1}]_{f g}=[\underbrace{t, \ldots, t}_{n+1}]_{f}[t]_{g}+\sum_{i=1}^{n}[\underbrace{t, \ldots, t}_{i}]_{f}[\underbrace{t, \ldots, t}_{n+2-i}]_{g}
$$

at $t \in \mathbb{R}$, which implies in turn that

$$
\begin{aligned}
& \frac{1}{n !}(f g)^{(n)}(t) \\
& =\frac{1}{n !} f^{(n)}(t) g(t) \\
& \quad+\sum_{i=1}^{n} \frac{1}{(i-1) !} f^{(i-1)}(t) \frac{1}{(n-i+1) !} g^{(n-i+1)}(t),
\end{aligned}
$$

and consequently

$$
\begin{aligned}
& (f g)^{(n)}(t) \\
& \quad=f^{(n)}(t) g(t)+\sum_{i=0}^{n-1}\left(\begin{array}{c}
n \\
i
\end{array}\right) f^{(i)}(t) g^{(n-i)}(t) \\
& \quad=\sum_{i=0}^{n}\left(\begin{array}{c}
n \\
i
\end{array}\right) f^{(i)}(t) g^{(n-i)}(t),
\end{aligned}
$$

where $f^{(0)}(t):=f(t), g^{(0)}(t):=g(t)$.

In view of the facts (57) and (60), we can prove the following result.
Theorem 11. Let $t \in \mathbb{R}$ and let $f(z) \in \mathcal{N}_{\kappa}$ have an integral representation of the form (10). Then for some $m \in \mathbb{N}^{+}$, the angular derivatives $f^{(k)}(t)(k=0,1, \ldots, 2 m-1)$ exist and are real numbers if and only if

$$
\int_{\mathbb{R}}(u-t)^{-2 m} \mathrm{~d} \sigma(\mathrm{u})<+\infty .
$$

In that case,

$$
f^{(k)}(t)=\angle \lim _{z \rightarrow t} f^{(k)}(z), \quad k=0,1, \ldots, 2 m-1 .
$$

Proof. Observe that the integrand in (10) can be rewritten as $z+\left(z^{2}+y_{0}^{2}\right)(u-z)^{-1}$, so the integral representation (10) of $f(z)$ can be rewritten as

$$
\begin{aligned}
f(z)= & \frac{1}{\prod_{j=1}^{\kappa_{1}}\left(z-\alpha_{j}\right)\left(z-\bar{\alpha}_{j}\right)} \\
& \times\left(\left(z^{2}+y_{0}^{2}\right)^{\kappa+1} \int_{\mathbb{R}} \frac{\mathrm{d} \sigma(u)}{u-z}+\sum_{j=0}^{2 \kappa+1} \widetilde{b}_{j} z^{j}\right),
\end{aligned}
$$

where $\widetilde{b}_{0}, \ldots, \widetilde{b}_{2 \kappa+1} \in \mathbb{R}$ with $\widetilde{b}_{2 \kappa+1} \geq 0$, and $\alpha_{j}, \kappa_{1}, y_{0}$, and $\sigma(u)$ are as in (10). Let now

$$
\begin{gathered}
s(z)=\left(z^{2}+y_{0}^{2}\right)^{\kappa+1}\left(\prod_{j=1}^{\kappa_{1}}\left(z-\alpha_{j}\right)\left(z-\bar{\alpha}_{j}\right)\right)^{-1}, \\
g(z)=\int_{\mathbb{R}} \frac{\mathrm{d} \sigma(u)}{u-z}, \\
r(z)=\left(\prod_{j=1}^{\kappa_{1}}\left(z-\alpha_{j}\right)\left(z-\bar{\alpha}_{j}\right)\right)^{-1} \sum_{j=0}^{2 \kappa+1} \widetilde{b}_{j} z^{j} .
\end{gathered}
$$

Then $f(z)=s(z) g(z)+r(z)$, or equivalently, $g(z)=$ $\widetilde{s}(z) f(z)+\widetilde{r}(z)$, where $\widetilde{s}(z)=s(z)^{-1}, \tilde{r}(z)=s(z)^{-1} r(z)$. Since, 
for real $t, s^{(k)}(t), r^{(k)}(t), \widetilde{s}^{(k)}(t), \widetilde{r}^{(k)}(t) \in \mathbb{R}$ and $s(t), \widetilde{s}(t)>0$, from (57) and (60), we deduce that the angular derivatives $f^{(k)}(t)(k=0,1, \ldots, 2 m-1)$ exist and are real numbers if and only if the angular derivatives $g^{(k)}(t)(k=0,1, \ldots, 2 m-1)$ exist and are real numbers. Thus, by Lemma 8 , the angular derivatives $f^{(k)}(t)(k=0,1, \ldots, 2 m-1)$ exist and are real numbers if and only if the inequality (61) holds.

From the relation $f(z)=s(z) g(z)+r(z)$, together with Proposition 9 and (57)-(60), it follows in turn that

$$
\begin{aligned}
& \angle \lim _{z \rightarrow t} f^{(k)}(z) \\
& \quad=\angle \lim _{z \rightarrow t}\left(\sum_{i=0}^{k}\left(\begin{array}{c}
k \\
i
\end{array}\right) s^{(k-i)}(z) g^{(i)}(z)+r^{(k)}(z)\right) \\
& \quad=\sum_{i=0}^{k}\left(\begin{array}{c}
k \\
i
\end{array}\right) s^{(k-i)}(t) g^{(i)}(t)+r^{(k)}(t) \\
& =f^{(k)}(t), \quad k=0,1, \ldots, 2 m-1 .
\end{aligned}
$$

In the sequel, we study the property of the asymptotic expansion of a function $f(z)$ in the class $\mathcal{N}_{\kappa}$ at a real point $t$.

Proposition 12. Let $f(z) \in \mathcal{N}_{\kappa}$ and $t \in \mathbb{R}$. If, for some $m \in$ $\mathbb{N}^{+}, f(z)$ has an asymptotic expansion at $t$ of the form:

$$
\begin{aligned}
f(z)= & h_{-1}+\sum_{i=0}^{2 m} h_{i}(z-t)^{i+1} \\
& +o\left((z-t)^{2 m+1}\right) \quad\left(z \in \pi_{\epsilon}(t), z \longrightarrow t\right),
\end{aligned}
$$

where $h_{i} \in \mathbb{R}(i=-1,0, \ldots, 2 m)$, then $f(z)$ has the angular derivatives of order from 1 to $2 m+1$ at the real point $t$; moreover,

$$
h_{i-1}=\frac{1}{i !} f^{(i)}(t), \quad i=0,1, \ldots, 2 m+1 .
$$

Proof. From the asymptotic expansion (66) of $f(z)$ at $z=t$, we deduce

$$
\begin{gathered}
{[t]_{f}=\angle \lim _{z \rightarrow t} f(z)=h_{-1},} \\
{[t, t]_{f}=\angle \lim _{z \rightarrow t} \frac{f(z)-h_{-1}}{z-t}=h_{0},} \\
{[t, t, t]_{f}=\angle \lim _{z \rightarrow t} \frac{[z, t]_{f}-[t, t]_{f}}{z-t}} \\
=\angle \lim _{z \rightarrow t} \frac{f(z)-h_{-1}-h_{0}(z-t)}{(z-t)^{2}}=h_{1},
\end{gathered}
$$

$$
\begin{aligned}
& {\left[\frac{t, \ldots, t}{2 m+2}\right]_{f}} \\
& =\angle \lim _{z \rightarrow t} \frac{[z, \overbrace{t, \ldots, t}^{2 m}]_{f}-\left[\frac{2 m+1}{t, \ldots, t}\right]_{f}}{z-t} \\
& =\angle \lim _{z \rightarrow t} \frac{f(z)-h_{-1}-h_{0}(z-t)-\cdots-h_{2 m-1}(z-t)^{2 m}}{(z-t)^{2 m+1}} \\
& =h_{2 m} .
\end{aligned}
$$

Observe that $f^{(i)}(t)=i ![\underbrace{t, \ldots, t}_{i+1}]_{f}, i=0,1, \ldots, 2 m+1$, and thus (67) holds.

Theorem 11 and Proposition 12 say actually that if, for some $m \in \mathbb{N}^{+}, f(z) \in \mathcal{N}_{\kappa}$ has an asymptotic expansion of the form (66) at $t \in \mathbb{R}$, then

$$
\angle \lim _{z \rightarrow t} f^{(i)}(z)=i ! h_{i-1}, \quad i=0,1, \ldots, 2 m+1
$$

Theorem 13. Let $f(z) \in \mathcal{N}_{\kappa}$ and $t \in \mathbb{R}$. If, for some $m \in \mathbb{N}^{+}$, $f(z)$ has an asymptotic expansion of the form (66) at $t \in \mathbb{R}$, then

$$
\begin{aligned}
& \lim _{y \rightarrow 0}[\underbrace{t+i y, \ldots, t+i y}_{k+1}, \underbrace{t-i y, \ldots, t-i y}_{l+1}]_{f} \\
& =h_{k+l}, \quad k, l=0,1, \ldots, m .
\end{aligned}
$$

Proof. Let $p(z)=h_{-1}+h_{0}(z-t)+\cdots+h_{2 m}(z-t)^{2 m+1}$ and $r(z)=f(z)-p(z)$. Then we have

$$
\begin{aligned}
\angle \lim _{z \rightarrow t} p^{(k)}(z) & =k ! h_{k-1} \\
& =\angle \lim _{z \rightarrow t} f^{(k)}(z), \quad k=0,1, \ldots, 2 m+1 .
\end{aligned}
$$

In view of the relation $f(z)=p(z)+r(z)$, we obtain, for real $y$,

$$
\begin{aligned}
& {[\underbrace{t+i y, \ldots, t+i y}_{k+1}, \underbrace{t-i y, \ldots, t-i y}_{l+1}]_{f}} \\
& =[\underbrace{t+i y, \ldots, t+i y}_{k+1}, \underbrace{t-i y, \ldots, t-i y}_{l+1}]_{p} \\
& +[\underbrace{t+i y, \ldots, t+i y, t-i y, \ldots, t-i y}_{k+1}]_{l+1}^{t-}
\end{aligned}
$$


Since $p(z)$ is a polynomial, we have

$$
\begin{aligned}
\lim _{y \rightarrow 0}[\underbrace{t+i y, \ldots, t+i y}_{k+1}, \underbrace{t-i y, \ldots, t-i y}_{l+1}]_{p} \\
=[\underbrace{t, t, \ldots, t}_{k+l+2}]_{p} \\
=\frac{1}{(k+l+1) !} p^{(k+l+1)}(t)=h_{k+l}, \quad 0 \leq k, l \leq m .
\end{aligned}
$$

Therefore, in order to prove (70), we need only to prove

$$
\begin{array}{r}
\lim _{y \rightarrow 0}[\underbrace{t+i y, \ldots, t+i y}_{k+1}, \underbrace{t-i y, \ldots, t-i y}_{l+1}]_{r}=0, \\
0 \leq k, l \leq m .
\end{array}
$$

By Proposition 3, we have

$$
\begin{gathered}
{[\underbrace{t+i y, \ldots, t+i y}_{k+1}, \underbrace{t-i y, \ldots, t-i y}_{l+1}]_{r}} \\
=\left.\frac{1}{k ! l !} \frac{\partial^{k+l}}{\partial \lambda^{k} \partial \mu^{l}}\left(\frac{r(\lambda)-r(\mu)}{\lambda-\mu}\right)\right|_{\lambda=t+i y, \mu=t-i y} \\
=\left.\frac{1}{k !} \frac{\mathrm{d}^{k}}{\mathrm{~d} \lambda^{k}} \frac{r(\lambda)}{(\lambda-t+i y)^{l+1}}\right|_{\lambda=t+i y} \\
+\left.\frac{1}{l !} \frac{\mathrm{d}^{l}}{\mathrm{~d} \mu^{l}} \frac{r(\mu)}{(\mu-t-i y)^{k+1}}\right|_{\mu=t-i y} .
\end{gathered}
$$

A direct computation leads to

$$
\begin{aligned}
& \left.\frac{1}{k !} \frac{\mathrm{d}^{k}}{\mathrm{~d} \lambda^{k}} \frac{r(\lambda)}{(\lambda-t+i y)^{l+1}}\right|_{\lambda=t+i y} \\
& =\sum_{s=0}^{k} \frac{(-1)^{s}(l+1) \cdots(l+s)}{s !(k-s) !} \frac{r^{(k-s)}(t+i y)}{(2 i y)^{l+s+1}} \\
& =\sum_{s=0}^{k} \frac{(-1)^{s}(l+1) \cdots(l+s)}{s !(k-s) !(2 i)^{l+s+1}} \\
& \quad \times \frac{\left(f^{(k-s)}(t+i y)-p^{(k-s)}(t+i y)\right)}{y^{l+s+1}} .
\end{aligned}
$$

By L'Hospital's rule, together with (69), we have

$$
\begin{aligned}
& \lim _{y \rightarrow 0} \frac{f^{(k-s)}(t+i y)-p^{(k-s)}(t+i y)}{y^{l+s+1}} \\
& =i \lim _{y \rightarrow 0} \frac{f^{(k-s+1)}(t+i y)-p^{(k-s+1)}(t+i y)}{(l+s+1) y^{l+s}} \\
& =i^{l+s+1} \lim _{y \rightarrow 0} \frac{f^{(k+l+1)}(t+i y)-p^{(k+l+1)}(t+i y)}{(l+s+1) !} \\
& =\frac{i^{l+s+1}}{(l+s+1) !} \lim _{y \rightarrow 0}\left(f^{(k+l+1)}(t+i y)-(k+l+1) ! h_{k+l}\right) \\
& =0 \quad(0 \leq k, l \leq m, 0 \leq s \leq k) .
\end{aligned}
$$

This means that

$$
\left.\lim _{y \rightarrow 0} \frac{1}{k !} \frac{\mathrm{d}^{k}}{\mathrm{~d} \lambda^{k}} \frac{r(\lambda)}{(\lambda-t+i y)^{l+1}}\right|_{\lambda=t+i y}=0, \quad 0 \leq k, l \leq m .
$$

In a similar way, we can verify that

$$
\left.\lim _{y \rightarrow 0} \frac{1}{l !} \frac{\mathrm{d}^{l}}{\mathrm{~d} \mu^{l}} \frac{r(\mu)}{(\mu-t-i y)^{k+1}}\right|_{\mu=t-i y}=0, \quad 0 \leq k, l \leq m .
$$

Then (74) holds, as needed.

From Theorems 7 and 13, we obtain an interesting result about the number of negative eigenvalues of the Hankel matrix constructed with the partial coefficients of the asymptotic expansion of $f(z) \in \mathcal{N}_{\kappa}$ at $t \in \mathbb{R}$.

Theorem 14. Let $f(z) \in \mathcal{N}_{\kappa}$ and $t \in \mathbb{R}$. If, for some $m \in \mathbb{N}^{+}$, $f(z)$ has an asymptotic expansion of the form (66) at real $t$, then $\nu(\mathbf{H}) \leq \kappa$, in which $\mathbf{H}:=\left(h_{k+l}\right)_{k, l=0}^{m}$.

Proof. By Theorem 13, we have

$$
\begin{aligned}
\lim _{y \rightarrow 0} P_{f} & (\underbrace{t+i y, \ldots, t+i y}_{m+1}) \\
& =\lim _{y \rightarrow 0}\left([\underbrace{t+i y, \ldots, t+i y}_{k+1}, \underbrace{t-i y, \ldots, t-i y}_{l+1}]_{f}\right)_{k, l=0}^{m} \\
& =\left(h_{k+l}\right)_{k, l=0}^{m}=\mathbf{H},
\end{aligned}
$$

and therefore $\mathbf{H}$ is a real Hankel matrix of order $m+$ 1. On the other hand, it follows from Theorem 7 that $\nu\left(P_{f}(\underbrace{t+i y, \ldots, t+i y}_{m+1})\right) \leq \kappa$. Since the eigenvalues of a matrix are continuous functions of its entries, we obtain $\nu(\mathbf{H}) \leq$ $\kappa$.

Finally, we consider two properties of the asymptotic expansion of $f(z) \in \mathcal{N}_{\kappa}$ at infinity. 
Theorem 15. If, for some $m \in \mathbb{N}^{+}, f(z) \in \mathcal{N}_{\kappa}$ has an asymptotic expansion at infinity:

$$
\begin{array}{r}
f(z)=-h_{-1}-\sum_{i=0}^{2 m} \frac{h_{i}}{z^{i+1}}+o\left(z^{-2 m-1}\right) \\
\left(z \in \pi_{\varepsilon}(0), z \longrightarrow \infty\right),
\end{array}
$$

where $h_{-1}, h_{0}, \ldots, h_{2 m} \in \mathbb{R}$, then $v(\mathbf{H}) \leq \kappa$, where $\mathbf{H}:=$ $\left(h_{k+l}\right)_{k, l=0}^{m}$.

Proof. Let $\tilde{f}(z)=f\left(-z^{-1}\right)$. Then $\tilde{f}(z) \in \mathcal{N}_{\kappa}$. It follows from (81) that $\widetilde{f}(z)$ has an asymptotic expansion at the point 0 of the form:

$$
\tilde{f}(z)=\widetilde{h}_{-1}+\widetilde{h}_{0} z+\widetilde{h}_{1} z^{2}+\cdots+\widetilde{h}_{2 m} z^{2 m+1}+o\left(z^{2 m+1}\right),
$$

where

$$
\widetilde{h}_{i}= \begin{cases}h_{i}, & \text { if } i \text { is even; } \\ -h_{i}, & \text { if } i \text { is odd }\end{cases}
$$

By Theorem 14, we obtain further that $v(\widetilde{\mathbf{H}}) \leq \kappa$, in which $\widetilde{\mathbf{H}}:=\left(\widetilde{h}_{k+l}\right)_{k, l=0}^{m}$. Observe that $\widetilde{\mathbf{H}}=\left(\widetilde{h}_{i+j}\right)_{i, j=0}^{m}=D \mathbf{H} D^{*}$, where $D=\operatorname{diag}\left(1,-1, \ldots,(-1)^{m}\right)$. Therefore, $\nu(\widetilde{\mathbf{H}})=v(\mathbf{H})$, so that $\nu(\mathbf{H}) \leq \kappa$, as desired.

Theorem 16. Let $f(z) \in \mathcal{N}_{\kappa}$ have an asymptotic expansion at infinity of the form:

$$
f(z)=-\sum_{i=0}^{2 n-2} \frac{h_{i}}{z^{i+1}}+o\left(z^{-2 n+1}\right) \quad\left(z \in \pi_{\varepsilon}(0), z \longrightarrow \infty\right),
$$

where $n \in \mathbb{N}^{+}, h_{0}, \ldots, h_{2 n-2} \in \mathbb{R}$, and let $\mathbf{H}:=\left(h_{i+j}\right)_{i, j=0}^{n-1}$. Then, for an arbitrary $m \in \mathbb{N}^{+}$and mutually different points $z_{1}, \ldots, z_{m} \in \mathbb{C}^{+} \cap D(f)$,

$$
v\left(L_{f}\left(z_{1}, \ldots, z_{m}\right)\right) \leq \kappa,
$$

where

$$
\begin{aligned}
& L_{f}\left(z_{1}, \ldots, z_{m}\right) \\
& :=\left(\begin{array}{c|ccc}
\mathbf{H} & C\left(z_{1}\right)^{*} & \ldots & C\left(z_{m}\right)^{*} \\
\hline C\left(z_{1}\right) & & & \\
\vdots & & P_{f}\left(z_{1}, \ldots, z_{m}\right) & \\
C\left(z_{m}\right) & &
\end{array}\right), \\
& C(z):=\left(f(z), z\left(f(z)+\frac{h_{0}}{z}\right), \ldots,\right. \\
& \left.z^{n-1}\left(f(z)+\frac{h_{0}}{z}+\frac{h_{1}}{z^{2}}+\cdots+\frac{h_{n-2}}{z^{n-1}}\right)\right) .
\end{aligned}
$$

Proof. Let $\widetilde{f}(z)=f\left(-z^{-1}\right)$. Then $\widetilde{f}(z) \in \mathcal{N}_{\kappa}$. For an arbitrary $m \in \mathbb{N}^{+}$and mutually different points $z_{1}, \ldots, z_{m} \in \mathbb{C}^{+} \cap D(f)$, $-z_{1}^{-1}, \ldots,-z_{m}^{-1} \in \mathbb{C}^{+} \cap D(\tilde{f})$ are also mutually different points. Then, for each $z \in \mathbb{C}^{+} \cap D(\tilde{f})$,

$$
\nu\left(P_{\widetilde{f}}(\underbrace{z, \ldots, z}_{n},-z_{1}^{-1}, \ldots,-z_{m}^{-1})\right) \leq \kappa .
$$

Observe that $\angle \lim _{z \rightarrow 0} P_{\tilde{f}}(\underbrace{z, \ldots, z}_{n},-z_{1}^{-1}, \ldots,-z_{m}^{-1})=P_{\widetilde{f}}(\underbrace{0, \ldots, 0}_{n}$, $\left.-z_{1}^{-1}, \ldots,-z_{m}^{-1}\right)$. Thus we infer

$$
\nu\left(P_{\widetilde{f}}(\underbrace{0, \ldots, 0}_{n},-z_{1}^{-1}, \ldots,-z_{m}^{-1})\right) \leq \kappa .
$$

On the other hand, it follows from (84) that

$$
\begin{aligned}
\tilde{f}(z)= & h_{0} z-h_{1} z^{2}+\cdots+h_{2 n-2} z^{2 n-1} \\
& +o\left(z^{2 n-1}\right) \quad\left(z \in \pi_{\epsilon}(0), z \longrightarrow 0\right) .
\end{aligned}
$$

Then we have

$$
\begin{aligned}
& P_{\tilde{f}}(\underbrace{0, \ldots, 0}_{n}) \\
& =\left((-1)^{i+j} h_{i+j}\right)_{i, j=0}^{n-1} \\
& =\operatorname{diag}\left(1,-1, \ldots,(-1)^{n-1}\right) \mathbf{H} \operatorname{diag}\left(1,-1, \ldots,(-1)^{n-1}\right)^{*},
\end{aligned}
$$

where $\mathbf{H}=\left(h_{i+j}\right)_{i, j=0}^{n-1}$ is a real Hankel matrix of order $n$. Choose $S:=\operatorname{diag}\left(1,-1, \ldots,(-1)^{n-1}, z_{1}^{-1}, \ldots, z_{m}^{-1}\right)$ which is a nonsingular matrix of order $n+m$. A direct computation leads to

$$
S P_{\tilde{f}}(\underbrace{0, \ldots, 0}_{n},-z_{1}^{-1}, \ldots,-z_{m}^{-1}) S^{*}=L_{f}\left(z_{1}, \ldots, z_{m}\right) .
$$

Therefore, $v\left(L_{f}\left(z_{1}, \ldots, z_{m}\right)\right)=v\left(P_{\widetilde{f}}(\underbrace{0, \ldots, 0}_{n},-z_{1}^{-1}, \ldots,-z_{m}^{-1})\right)$ $\leq \kappa$

\section{An Explicit Formula of the Negative Index of Real Rational Functions}

In this section, we give an explicit formula of the negative index of real rational functions by decomposing each real rational function into the sum of a real polynomial and a strictly proper real rational function.

From definition of a generalized Nevanlinna function, we can prove that each real polynomial is a generalized Nevanlinna function in the class $\mathcal{N}_{\kappa}$ with some negative index $\kappa$ determined by the following lemma (see, e.g., [1], [4, Section 3]).

Lemma 17. Let $p(z)=p_{0}+p_{1} z+\cdots+p_{m} z^{m} \quad\left(p_{m} \neq 0\right)$ be a real polynomial. Then $p(z) \in \mathcal{N}_{\kappa}$, where

$$
\kappa= \begin{cases}n, & \text { if } m=2 n+1, p_{m}>0 \\ n+1, & \text { if } m=2 n+1, p_{m}<0 \\ n, & \text { if } m=2 n .\end{cases}
$$


Proof. Obviously, the real polynomial $p(z)$ is holomorphic in $\mathbb{C}$ such that $p(\bar{z})=\overline{p(z)}$. Then $p(z)$ has no poles in $\mathbb{C}^{+}$and no generalized poles of nonpositive type in $\mathbb{R}$. In what follows we will determine the multiplicity of the point $\infty$ as a generalized pole of nonpositive type of $p(z)$.

(1) If $m=2 n+1$ and $p_{m}>0$, then

$$
\angle \lim _{z \rightarrow \infty} z^{-2 n-1} p(z)=p_{m}>0, \quad \angle \lim _{z \rightarrow \infty} z^{-2 n+1} p(z)=\infty,
$$

so that, by definition, the point $\infty$ is a generalized pole of nonpositive type of $p(z)$ with multiplicity $n$.

(2) If $m=2 n+1$ and $p_{m}<0$, then

$$
\begin{gathered}
\angle \lim _{z \rightarrow \infty} z^{-2(n+1)-1} p(z)=0, \\
\angle \lim _{z \rightarrow \infty} z^{-2(n+1)+1} p(z)=p_{m}<0,
\end{gathered}
$$

so that, by definition, the point $\infty$ is a generalized pole of nonpositive type of $p(z)$ with multiplicity $n+1$.

(3) If $m=2 n$, then

$$
\angle \lim _{z \rightarrow \infty} z^{-2 n-1} p(z)=0, \quad \angle \lim _{z \rightarrow \infty} z^{-2 n+1} p(z)=\infty,
$$

so that, by definition, the point $\infty$ is a generalized pole of nonpositive type of $p(z)$ with multiplicity $n$.

Since the total multiplicity of the poles of $p(z)$ in $\mathbb{C}^{+}$and the generalized poles of nonpositive type of $p(z)$ in $\mathbb{R} \cup\{\infty\}$ is equal to $\kappa$, the multiplicity of the point $\infty$ as a generalized pole of nonpositive type of $p(z)$ is equal to $\kappa$. Hence the equality (92) holds.

Let $f_{1}(z) \in \mathcal{N}_{\kappa_{1}}$ and $f_{2}(z) \in \mathcal{N}_{\kappa_{2}}$. Daho and Langer [9, Proposition 3.2] proposed a sufficient condition for $f_{1}(z)+$ $f_{2}(z) \in \mathcal{N}_{\kappa_{1}+\kappa_{2}}$ mentioned in introduction part. In view of this result, we can obtain a modified version of that sufficient condition (see also [1, Satz 1.13]).

Lemma 18. Let $f_{1}(z) \in \mathcal{N}_{\kappa_{1}}$ and $f_{2}(z) \in \mathcal{N}_{\kappa_{2}}$ have no common poles in $\mathbb{C}^{+}$and no common generalized poles of nompositive type in $\mathbb{R} \cup\{\infty\}$ and satisfy the following two conditions:

(1) if $\alpha \in \mathbb{R}$ is a generalized pole of nonpositive type of $f_{i}(z)(i=1,2)$, then $\angle \lim _{z \rightarrow \alpha}(z-\alpha) f_{j}(z)=0, j \neq i$;

(2) if the point $\infty$ is a generalized pole of nonpositive type of $f_{i}(z)(i=1,2)$, then $\angle \lim _{z \rightarrow \infty} z^{-1} f_{j}(z)=0, j \neq i$.

Then $f(z):=f_{1}(z)+f_{2}(z) \in \mathcal{N}_{\kappa_{1}+\kappa_{2}}$. Moreover, the poles of $f_{i}(z) \quad(i=1,2)$ in $\mathbb{C}^{+}$and the generalized poles of nonpositive type of $f_{i}(z) \quad(i=1,2)$ in $\mathbb{R} \cup\{\infty\}$ are also the poles of $f(z)$ in $\mathbb{C}^{+}$and the generalized poles of nonpositive type of $f(z)$ in $\mathbb{R} \cup\{\infty\}$ with the same multiplicities.
Proof. Without loss of generality, we need only to verify that Lemma 18 is valid in the case of $i=1$ and $j=2$. If $\alpha \in \mathbb{C}^{+}$is a pole of $f_{1}(z)$ of multiplicity $\pi$, then $f_{2}(z)$ is holomorphic at $\alpha$, and hence $\alpha$ is a pole of $f(z)=f_{1}(z)+f_{2}(z)$ of multiplicity $\pi$.

If $\alpha \in \mathbb{R}$ is a generalized pole of nonpositive type of $f_{1}(z)$ with multiplicity $\pi$, then $\angle \lim _{z \rightarrow \alpha}(z-\alpha)^{2 \pi-1} f_{1}(z)>0$ or is equal to $\infty$ and $\angle \lim _{z \rightarrow \beta}(z-\alpha)^{2 \pi+1} f_{1}(z) \leq 0$. It follows from condition (1) that

$$
\begin{aligned}
& \angle \lim _{z \rightarrow \alpha}(z-\alpha)^{2 \pi-1} f(z) \\
& \quad=\angle \lim _{z \rightarrow \alpha}(z-\alpha)^{2 \pi-1}\left(f_{1}(z)+f_{2}(z)\right) \\
& \quad>0 \text { or is equal to } \infty, \\
& \angle \lim _{z \rightarrow \alpha}(z-\alpha)^{2 \pi+1} f(z) \\
& \quad=\angle \lim _{z \rightarrow \beta}(z-\alpha)^{2 \pi+1}\left(f_{1}(z)+f_{2}(z)\right) \leq 0 .
\end{aligned}
$$

Thus, by definition, $\alpha \in \mathbb{R}$ is a generalized pole of nonpositive type of $f(z)=f_{1}(z)+f_{2}(z)$ with multiplicity $\pi$.

If the point $\infty$ is a generalized pole of nonpositive type of $f_{1}(z)$ with multiplicity $\pi$, then $\angle \lim _{z \rightarrow \infty} z^{-2 \pi+1} f_{1}(z)<0$ or is equal to $\infty$ and $\angle \lim _{z \rightarrow \infty} z^{-2 \pi-1} f_{1}(z) \geq 0$. It follows from condition (2) that

$$
\begin{gathered}
\angle \lim _{z \rightarrow \infty} z^{-2 \pi+1} f(z) \\
=\angle \lim _{z \rightarrow \infty} z^{-2 \pi+1}\left(f_{1}(z)+f_{2}(z)\right) \\
<0 \text { or is equal to } \infty, \\
\angle \lim _{z \rightarrow \infty} z^{-2 \pi-1} f(z)=\angle \lim _{z \rightarrow \infty} z^{-2 \pi-1}\left(f_{1}(z)+f_{2}(z)\right) \geq 0 .
\end{gathered}
$$

Thus the point $\infty$ is a generalized pole of nonpositive type of $f(z)=f_{1}(z)+f_{2}(z)$ with multiplicity $\pi$ by definition.

The above analysis shows that the poles of $f_{i}(z)(i=$ $1,2)$ in $\mathbb{C}^{+}$and the generalized poles of nonpositive type of $f_{i}(z)(i=1,2)$ in $\mathbb{R} \cup\{\infty\}$ are also the poles of $f(z)$ in $\mathbb{C}^{+}$and the generalized poles of nonpositive type of $f(z)$ in $\mathbb{R} \cup\{\infty\}$ with the same multiplicities. However, since $f_{1}(z)$ and $f_{2}(z)$ have no common poles in $\mathbb{C}^{+}$and no common generalized poles in $\mathbb{R} \cup\{\infty\}$, the total multiplicity of poles of $f(z)$ in $\mathbb{C}^{+}$and the generalized poles of nonpositive type of $f(z)$ in $\mathbb{R} \cup\{\infty\}$ is greater than or equal to $\kappa_{1}+\kappa_{2}$, and hence $f(z) \in \mathcal{N}_{\kappa}$ with $\kappa \geq \kappa_{1}+\kappa_{2}$. On the other hand, as stated in the introduction part, we have $\kappa \leq \kappa_{1}+\kappa_{2}$. Thus $\kappa=\kappa_{1}+\kappa_{2}$.

To describe the negative index of a strictly proper real rational function, we have to consider the Bézout matrix of a pair of polynomials (see, e.g., [17-19]) and the generating functions of a Hankel matrix.

Let $f(z)$ and $g(z)$ be real polynomials with $\max \{\operatorname{deg} f(z), \operatorname{deg} g(z)\}=n$. We define the Bézout matrix 
of polynomials $f(z)$ and $g(z)$ as the $n$ by $n$ matrix $B(f, g):=\left(b_{i j}\right)_{i, j=0}^{n-1}$ generated by

$$
\frac{f(z) g(\omega)-f(\omega) g(z)}{z-\omega}=\sum_{i, j=0}^{n-1} b_{i j} z^{i} \omega^{j}
$$

Clearly, we have that $B(f, g)=-B(g, f)$ and $B(f, g)=$ $B(f, g)^{\mathrm{T}}$.

Let $h_{0}, h_{1}, \ldots, h_{2 n-2} \in \mathbb{R}$ be given. Let $f(z)$ be a real polynomial of degree $l$, and let $g(z)$ be a real polynomial of degree not more than $(l-1)$ such that $\operatorname{gcd}(f(z), g(z))=$ 1. If the strictly proper rational function $g(z) / f(z)$ has an asymptotic expansion at the point $\infty$ as follows:

$$
\frac{g(z)}{f(z)}=\frac{h_{0}}{z}+\frac{h_{1}}{z^{2}}+\cdots+\frac{h_{2 n-2}}{z^{2 n-1}}+o\left(z^{-2 n+1}\right) \quad(z \longrightarrow \infty),
$$

then $g(z) / f(z)$ is called a generating function, of McMillan degree $l$, of the Hankel matrix $\mathbf{H}:=\left(h_{i+j}\right)_{i, j=0}^{n-1}$, and in this case that Hankel matrix $\mathbf{H}$ is also represented as $\mathbf{H}=\mathbf{H}_{n}(g / f)$.

It is well-known that the Bézout matrix and the Hankel matrix have the following relation; see, for example, $[17,20]$.

Lemma 19. Let $f(z)=\sum_{i=0}^{n} f_{i} z^{i}\left(f_{n} \neq 0\right)$ and $g(z)=$ $\sum_{i=0}^{n} g_{i} z^{i}$ be two real polynomials such that the rational function $g(z) / f(z)$ has an asymptotic expansion at the point $\infty$ as

$$
\frac{g(z)}{f(z)}=h_{-1}+\frac{h_{0}}{z}+\frac{h_{1}}{z^{2}}+\cdots+\frac{h_{2 n-2}}{z^{2 n-1}}+o\left(z^{-2 n+1}\right) .
$$

Then

$$
B(f, g)=S(f) \mathbf{H}_{n}\left(\frac{g}{f}\right) S(f)
$$

where

$$
S(f):=\left(\begin{array}{cccc}
f_{1} & f_{2} & \cdots & f_{n} \\
f_{2} & & . & \\
\vdots & . & & \\
f_{n} & & & 0
\end{array}\right), \quad \mathbf{H}_{n}\left(\frac{g}{f}\right)=\left(h_{i+j}\right)_{i, j=0}^{n-1} .
$$

In the following, we give the result on the negative index of a strictly proper real rational function and its detailed proof.

Theorem 20. Let $f(z)$ be a real polynomial of degree $n$, and let $g(z)$ be a real polynomial of degree not more than $(n-1)$. If $r(z):=g(z) / f(z)$ has an asymptotic expansion at the point $\infty$ as follows:

$$
\begin{aligned}
r(z)=\frac{g(z)}{f(z)}= & -\frac{h_{0}}{z}-\frac{h_{1}}{z^{2}} \\
& -\cdots-\frac{h_{2 n-2}}{z^{2 n-1}}+o\left(z^{-2 n+1}\right) \quad(z \longrightarrow \infty),
\end{aligned}
$$

then $r(z) \in \mathcal{N}_{\kappa}$, where $\kappa=\nu\left(\mathbf{H}_{n}(-g / f)\right)=\nu(\mathbf{H})$ and $\mathbf{H}:=$ $\left(h_{i+j}\right)_{i, j=0}^{n-1}$.
Proof. Observe that $r(\bar{z})=\overline{r(z)}$ and, for an arbitrary $m \in \mathbb{N}^{+}$ and distinct points $z_{1}, \ldots, z_{m} \in \mathbb{C}^{+} \cap D(r)$,

$$
\begin{aligned}
& P_{r}\left(z_{1}, \ldots, z_{m}\right) \\
& =\left(\frac{r\left(z_{i}\right)-r\left(\bar{z}_{j}\right)}{z_{i}-\bar{z}_{j}}\right)_{i, j=1}^{m} \\
& =\left(\frac{g\left(z_{i}\right) / f\left(z_{i}\right)-g\left(\bar{z}_{j}\right) / f\left(\bar{z}_{j}\right)}{z_{i}-\bar{z}_{j}}\right)_{i, j=1}^{m} \\
& =\operatorname{diag}\left(f\left(z_{1}\right)^{-1}, \ldots, f\left(z_{m}\right)^{-1}\right) V\left(z_{1}, \ldots, z_{m}\right) \\
& \quad \times B(g, f) V\left(z_{1}, \ldots, z_{m}\right)^{*} \\
& \quad \times \operatorname{diag}\left(f\left(z_{1}\right)^{-1}, \ldots, f\left(z_{m}\right)^{-1}\right)^{*},
\end{aligned}
$$

where

$$
V\left(z_{1}, \ldots, z_{m}\right)=\left(\begin{array}{cccc}
1 & z_{1} & \cdots & z_{1}^{n-1} \\
\vdots & \vdots & & \vdots \\
1 & z_{m} & \cdots & z_{m}^{n-1}
\end{array}\right)
$$

Thus we obtain that $\nu\left(P_{r}\left(z_{1}, \ldots, z_{m}\right)\right) \leq \nu(B(g, f))$, and hence $r(z) \in \mathcal{N}_{\kappa}$ and $\kappa \leq \nu(B(g, f))$.

On the other hand, since $r(z)$ has an asymptotic expansion of the form (103), by Theorem 15, we have $r(z) \in \mathcal{N}_{\kappa}$, with $\kappa \geq \nu(\mathbf{H})$, where $\mathbf{H}=\left(h_{i+j}\right)_{i, j=0}^{n-1}$. Moreover, Lemma 19 provides that $B(f, g)=S(f) \mathbf{H}_{n}(g / f) S(f)=-S(f) \mathbf{H} S(f)^{*}$, where $S(f)$ is invertible, so that $B(g, f)=S(f) \mathbf{H} S(f)^{*}$, and hence $\nu(B(g, f))=\nu(\mathbf{H}) \leq \kappa$. Therefore, we have $r(z) \in \mathcal{N}_{\kappa}$ with $\kappa=\nu(\mathbf{H})$.

For an arbitrary real rational function $R(z):=g(z) / f(z)$, where $g(z):=\sum_{i=0}^{m} g_{i} z^{i}\left(g_{m} \neq 0\right)$ and $f(z):=\sum_{j=0}^{n} f_{j} z^{j}\left(f_{n}=\right.$ 1) are real polynomials such that $\operatorname{gcd}(f(z), g(z))=1$ and $m, n \in \mathbb{N}^{+}$. By the Euclidean division of polynomials, $g(z)$ has an expression of the form $g(z)=f(z) q(z)+r(z)$, in which $r(z)=0$ or $\operatorname{deg} r(z)<\operatorname{deg} f(z)$; hence, we have $R(z)=$ $g(z) / f(z)=q(z)+r(z) / f(z)$, where $q(z)=0$ (if $m<n$ ) or $q(z)$ is the form $q(z)=\sum_{i=0}^{m-n} q_{i} z^{i}$ (if $m \geq n$ ), with $q_{m-n}=g_{m}$.

If $m<n$, then, by Theorem 20 , the negative index of $R(z)$ is equal to the negative inertia index of the $n$ by $n$ Hankel matrix generated by the function $-R(z)$.

If $m \geq n$, we define $q(z)$ as the polynomial part of $R(z)$ and $r(z) / f(z)$ as the strictly proper rational function part of $R(z)$. In this case, the negative index of $q(z)$ is given by use of Lemma 17 and the negative index of $r(z) / f(z)$ is derived by use of Theorem 20 . Therefore, by Lemma 18, the negative index of a real rational function $R(z)$ is the sum of the negative indexes of $q(z)$ and $r(z) / f(z)$. Thereby, we obtain an explicit formula of the negative index of real rational functions.

Theorem 21. Let $R(z)=g(z) / f(z)$ be a real rational function, where $g(z)=\sum_{i=0}^{m} g_{i} z^{i}\left(g_{m} \neq 0\right)$ and $f(z)=\sum_{j=0}^{n} f_{j} z^{j}\left(f_{n}=1\right)$ are real polynomials with $g c d(f(z), g(z))=1$ and $m, n \in \mathbb{N}^{+}$. Then 
(1) If $m<n$, then $R(z) \in \mathcal{N}_{\kappa}$, where $\kappa=\nu\left(\mathbf{H}_{n}(-R)\right)$.

(2) If $m \geq n$, then $R(z) \in \mathcal{N}_{\kappa_{1}+\kappa_{2}}$, where

$$
\begin{aligned}
& \kappa_{1}= \begin{cases}l, & \text { if } m-n=2 l+1, g_{m}>0 ; \\
l+1, & \text { if } m-n=2 l+1, g_{m}<0 ; \\
l, & \text { if } m-n=2 l,\end{cases} \\
& \kappa_{2}=v\left(\mathbf{H}_{n}\left(-\frac{r}{f}\right)\right),
\end{aligned}
$$

where $r(z) / f(z)$ is the strictly proper rational function part of $R(z)$.

\section{Conclusion}

In this paper, we consider some propositions on generalized Nevanlinna functions with detailed proofs. We prove that the Hermitian matrix $\left(\left[z_{i}, \bar{z}_{j}\right]_{f}\right)_{i, j=1}^{m}$ is congruent to the Hermitian matrix $\left(\left[z_{1}, \ldots, z_{i}, \bar{z}_{1}, \ldots, \bar{z}_{j}\right]_{f}\right)_{i, j=1}^{m}$, given in (29). This result is quite important in the proof of an equivalent definition of a generalized Nevanlinna function (see Theorem 7), because it can be used to verify the fact that the negative inertia index of every Hermitian generalized Loewner matrix generated by a generalized Nevanlinna function of the class $\mathcal{N}_{\kappa}$ does not exceed $\kappa$. We also obtain that the negative inertia index of the Hankel matrix constructed with the partial coefficients of the asymptotic expansions of a generalized Nevanlinna function of the class $\mathcal{N}_{\kappa}$ at a real point or at infinity is less than or equal to $\kappa$, and the same result holds also for the Loewner matrix $L_{f}\left(z_{1}, \ldots, z_{m}\right)$ defined by (86). Finally, based on the relations among Loewner, Bézout, and Hankel matrices, we give an explicit formula of the negative index of real rational functions. These results stated in this paper will be applied to solve the indefinite Hamburger moment problems and the multiple NevanlinnaPick interpolation problems for the class $\mathcal{N}_{\kappa}$ in subsequent work.

\section{Conflict of Interests}

The authors declare that there is no conflict of interests regarding the publication of this paper.

\section{Acknowledgments}

This work was supported by the National Natural Science Foundation of China (nos. 11071017 and 11271045) and the Program for New Century Excellent Talents in University.

\section{References}

[1] M. G. Krein and H. Langer, "Über einige Fortsetzungsprobleme, die eng mit der Theorie hermitescher Operatoren im Raume $\mathrm{II}_{k}$ zusammenhangen. I. Einige Funktionenklassen und ihre Darstellungen," Mathematische Nachrichten, vol. 77, pp. 187236, 1977.

[2] N. I. Akhiezer, The Classical Moment Problem and Some Related Questions in Analysis, Oliver and Boyd, London, UK, 1965.
[3] M. G. Krein and A. A. Nudelman, The Markov Moment and Extremal Problems, vol. 50 of Translations of Mathematical Monographs, American Mathematical Society, Providence, RI, USA, 1977.

[4] M. Derevyagin, "On the Schur algorithm for indefinite moment problem," Methods of Functional Analysis and Topology, vol. 9, no. 2, pp. 133-145, 2003.

[5] V. Derkach, S. Hassi, and H. de Snoo, "Truncated moment problems in the class of generalized Nevanlinna functions," Mathematische Nachrichten, vol. 285, no. 14-15, pp. 1741-1769, 2012.

[6] A. Dijksma, H. Langer, A. Luger, and Y. Shondin, "A factorization result for generalized Nevanlinna functions of the class $N_{k}$," Integral Equations and Operator Theory, vol. 36, no. 1, pp. 121$125,2000$.

[7] M. G. Krein and H. Langer, "Some propositions on analytic matrix functions related to the theory of operators in the space $I I_{k}$," Acta Scientiarum Mathematicarum, vol. 43, no. 1-2, pp. 181205, 1981.

[8] H. Langer, "A characterization of generalized zeros of negative type of functions of the class $N_{k}$," Operator Theory: Advances and Applications, vol. 17, pp. 201-212, 1986.

[9] K. Daho and H. Langer, "Matrix functions of the class $N \kappa$," Mathematische Nachrichten, vol. 120, pp. 275-294, 1985.

[10] V. Derkach, S. Hassi, and H. de Snoo, "Generalized Nevanlinna functions with polynomial asymptotic behaviour at infinity and regular perturbations," in Operator Theory and Analysis, vol. 122 of Operator Theory: Advances and Applications, pp. 169-189, Birkhäuser, 2001.

[11] V. Derkach, S. Hassi, and H. de Snoo, "Asymptotic expansions of generalized Nevanlinna functions and their spectral properties," in Operator Theory: Advances and Applications, vol. 175, pp. 51-88, 2007.

[12] S. Hassi and A. Luger, "Generalized zeros and poles of $N_{k^{-}}$ functions: on the underlying spectral structure," Methods of Functional Analysis and Topology, vol. 12, no. 2, pp. 131-150, 2006.

[13] M. G. Krein and H. Langer, "Über die Q-funktion eines $\Pi$-hermitischen operators im raume $\Pi_{k}$," Acta Scientiarum Mathematicarum (Szeged), vol. 34, pp. 191-230, 1973.

[14] G. N. Chen and H. P. Zhang, "More on Loewner matrices," Linear Algebra and its Applications, vol. 203-204, pp. 265-300, 1994.

[15] M. Fiedler, "Hankel and Loewner matrices," Linear Algebra and its Applications, vol. 58, pp. 75-95, 1984.

[16] G.-N. Chen and Y.-J. Hu, "Multiple Nevanlinna-Pick interpolation with both interior and boundary data and its connection with the power moment problem," Linear Algebra and its Applications, vol. 323, no. 1-3, pp. 167-194, 2001.

[17] G.-N. Chen, Matrix Theory and Its Applications, Science Press, Beijing, China, 2nd edition, 2007.

[18] M. Fiedler and V. Pták, "Bézoutians and intertwining matrices," Linear Algebra and Its Applications, vol. 86, pp. 43-51, 1987.

[19] P. Lancaster and M. Tismenetsky, The Theory of Matrices: With Applications, American Academic Press, New York, NY, USA, 2nd edition, 1985.

[20] G. Heinig and K. Rost, Algebraic Methods for Toeplitz-like Matrices and Operators, Birkhäuser, Boston, Mass, USA, 1984. 


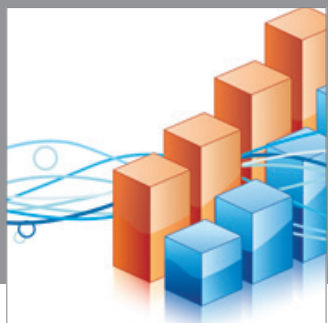

Advances in

Operations Research

mansans

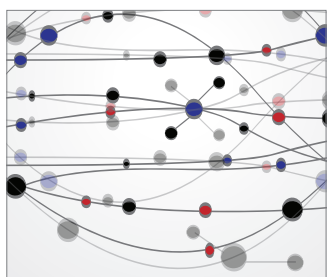

The Scientific World Journal
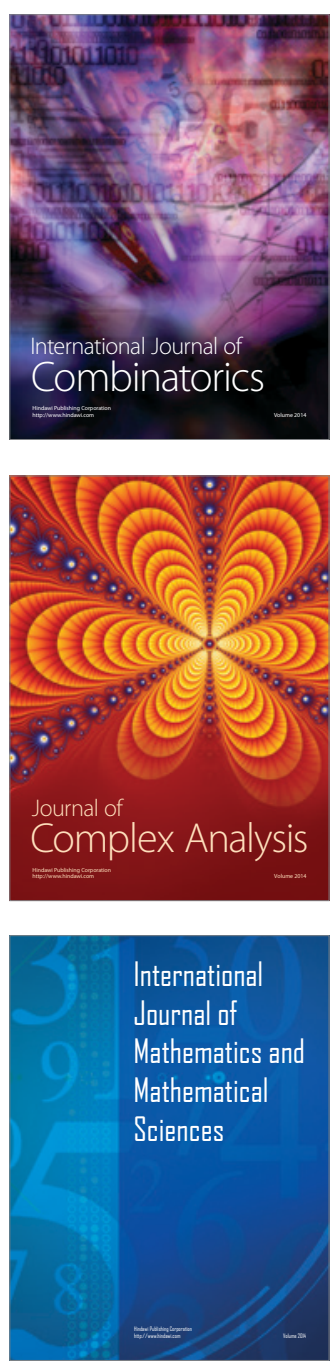
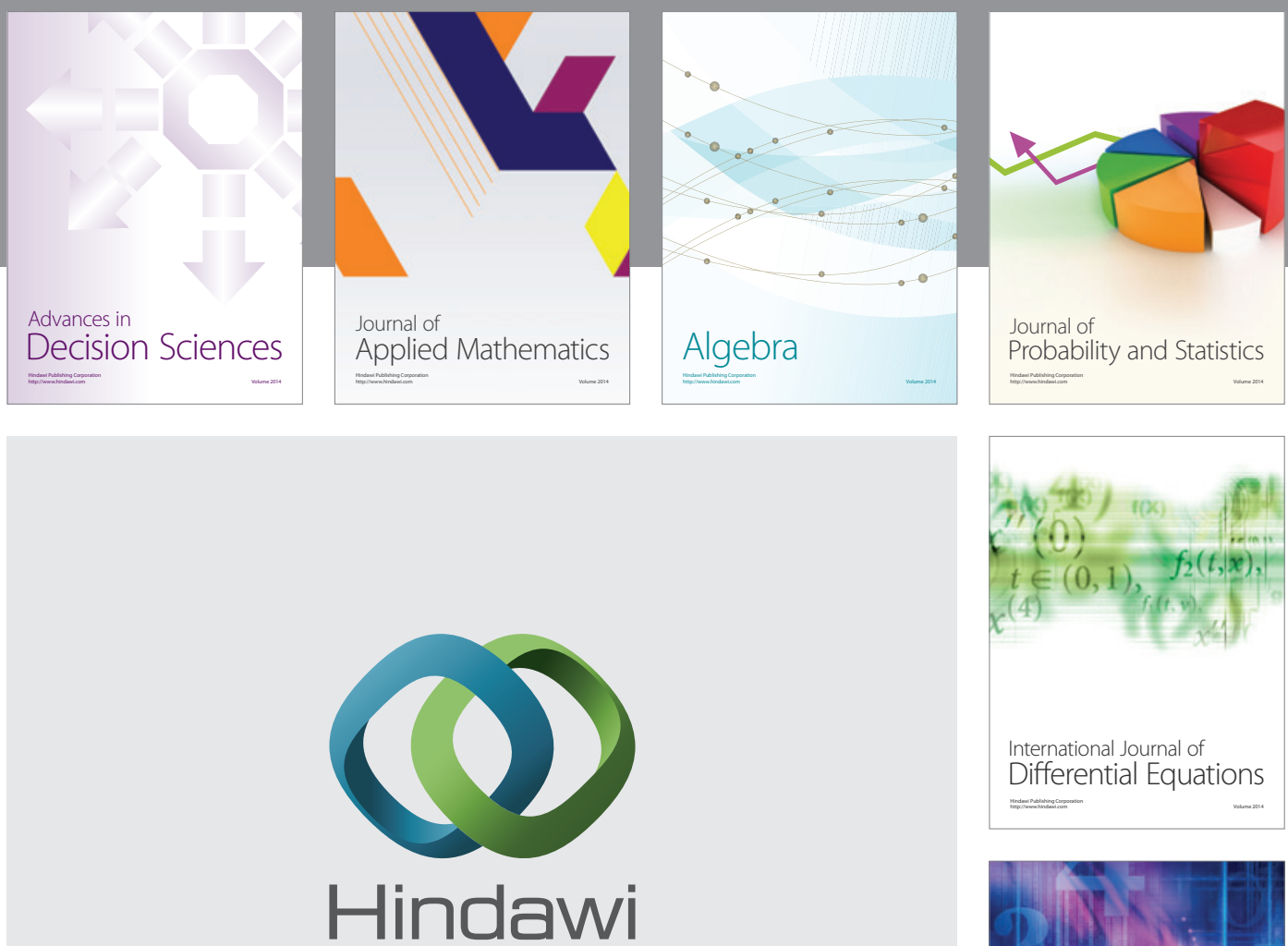

Submit your manuscripts at http://www.hindawi.com
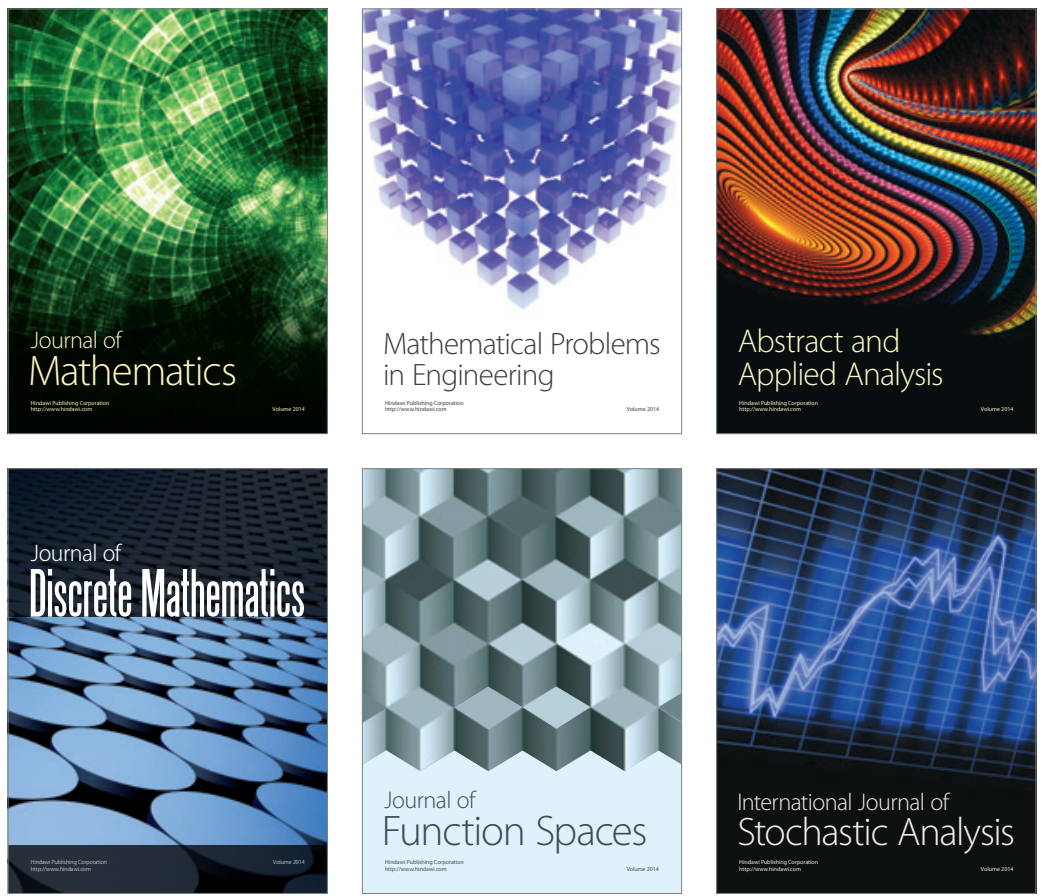

Journal of

Function Spaces

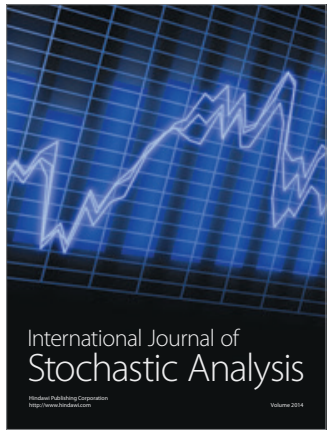

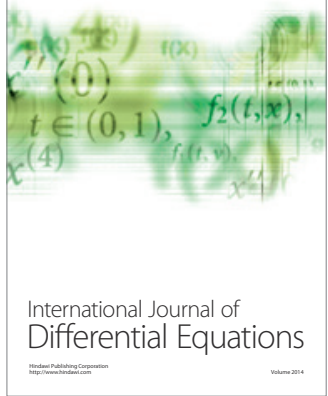
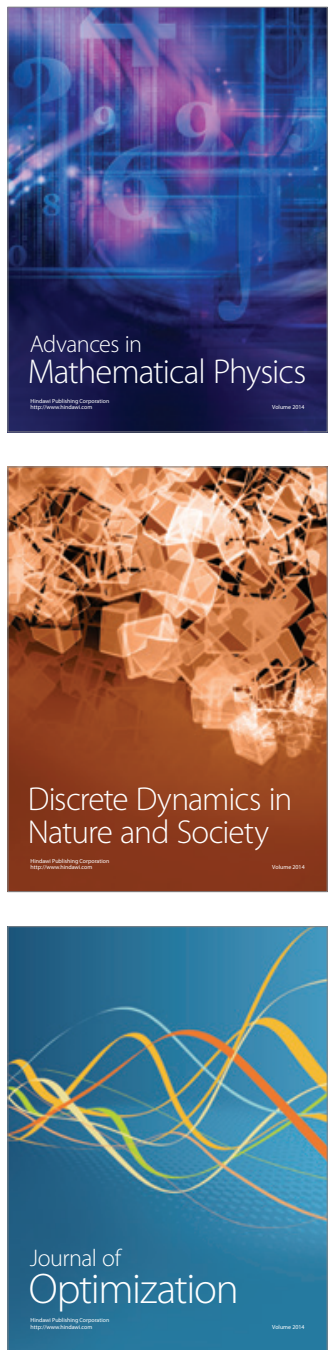